\title{
THE RIEMANN PROBLEM FOR A SIMPLE MODEL OF PHASE TRANSITION*
}

\author{
EDWIGE GODLEWSKI ${ }^{\dagger}$ AND NICOLAS SEGUIN ${ }^{\ddagger}$
}

\begin{abstract}
We consider in this paper a simple model of liquid-vapor phase transition. The PDE system corresponds to the isothermal $p$-system and the pressure law is defined for all positive density and involves a constant zone. Such a pressure law can occur for instance when considering the Van der Waals equation of state complemented by the Maxwell's law. The Riemann problem is globally solved for all initial states, as well in the pure phases as in the mixture zone.
\end{abstract}

Key words. systems of conservation laws, Riemann problem, phase transition, resonance, pressureless gas.

AMS subject classifications. $\quad 35 \mathrm{~L} 65,35 \mathrm{~L} 80,76 \mathrm{~T} 10$

\section{Introduction}

The model we consider is the isothermal $p$-system. It is composed by the system of partial differential equations

$$
\begin{aligned}
& \partial_{t} \tau-\partial_{x} u=0, \\
& \partial_{t} u+\partial_{x} p=0
\end{aligned}
$$

where $\tau$ denotes the specific volume, $u$ the velocity and $p$ the pressure of the fluid. All these unknowns depend on the time $t$ and the Lagrangian variable $x,(t, x) \in \mathbb{R}_{+} \times \mathbb{R}$. We also add the pressure law

$$
p=\mathscr{P}(\tau)
$$

We then define $\mathbf{u}=(\tau, u)^{T} \in \Omega=\mathbb{R}_{+}^{*} \times \mathbb{R}$ and $\mathbf{f}(\mathbf{u})=(-u, \mathscr{P}(\tau))^{T}(\Omega$ will be referred to as the phase space).

All the thermodynamic behavior of the fluid, in particular the transition between the liquid and the vapor states, is contained in the law (1.3). We can refer for instance to the Van der Waals equation of state (see the solid line in Fig. 1.1). The difficulty is to cope with the elliptic zone defined by the specific volumes which verify $\mathscr{P}^{\prime}(\tau)>0$. Indeed, system $(1.1-1.3)$ is unstable in this zone (the sound speed $c(\mathbf{u})=\sqrt{-\mathscr{P}^{\prime}(\tau)}$ becomes complex). Numerous works deal with this problem, see for instance $[2,19]$ and references therein.

The model we focus on may be seen as a Van der Waals type equation of state complemented with Maxwell's law. Roughly speaking, Maxwell's law introduces two constant specific volumes $0<\tau_{2}^{*}<\tau_{1}^{*}$ which are given by the thermodynamic equilibrium and limit the mixture zone (see Fig. 1.1). Assuming that the pressure is constant in the mixture zone, the Van der Waals pressure law is replaced by a straight line in $\left[\tau_{2}^{*}, \tau_{1}^{*}\right]$, depicted by the dashed line in Fig. 1.1 .

${ }^{*}$ Received: November 21, 2005; accepted (in revised version): January 30, 2006. Communicated by Shi Jin.

†Université Pierre et Marie Curie - Paris 6, UMR 7598 LJLL, Paris, F-75005, France (godlewski@ ann.jussieu.fr).

¥Université Pierre et Marie Curie - Paris 6, UMR 7598 LJLL, Paris, F-75005, France (seguin@ ann.jussieu.fr). 


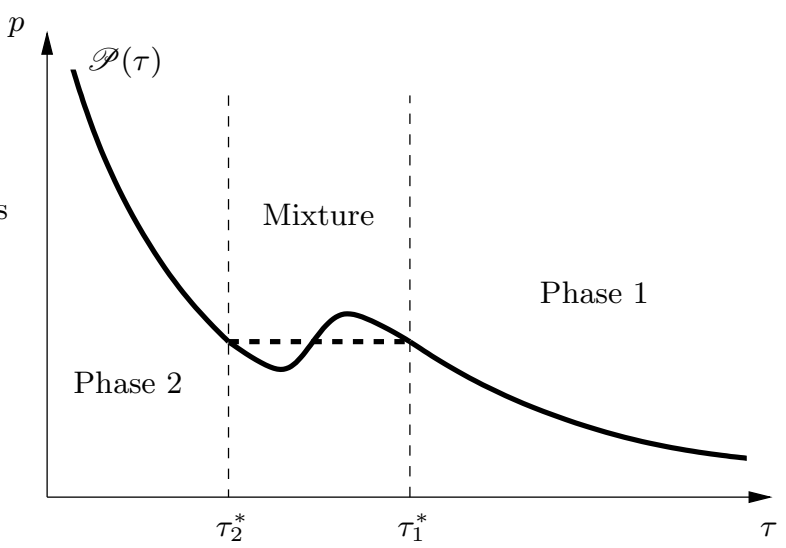

FIG. 1.1. Van der Waals pressure law and Maxwell closure law.

As a consequence, the pressure law is now globally nonincreasing and its derivative (thus the sound speed) vanishes in the mixture zone. Note that a viscous regularization of (1.1-1.3) has been studied by Roytburd and Slemrod in [25] with the help of compensated compactness and equivalence of entropy criteria. The Riemann problem with data of small amplitude has been investigated by Roytburd in [24]. Another model has been studied by Shearer in [27], based on the Van der Waals equation of state for which all stationary waves of phase transition are admissible. Here, the physical model is slightly different since only stationary waves of phase transition satisfying Maxwell's law are allowed, which results in a more constraining problem with a pressure law which is only piecewise $\mathscr{C}^{1}$ and thus leads to different solutions.

The present model is very simple (in particular, it does not include any nonclassical theory [19]) and it is suitable for numerical approximations since the phase space is convex. A particular model of pressure law which falls within the present framework is the one studied by Caro et al. [5]. Assuming that the two phases behave as isothermal perfect gases, the pressure law of the mixture is

$$
\mathscr{P}(\tau)= \begin{cases}a_{2} / \tau & \text { if } 0<\tau<\tau_{2}^{*}, \\ a_{2} / \tau_{2}^{*} & \text { if } \tau_{2}^{*} \leq \tau \leq \tau_{1}^{*}, \\ a_{1} / \tau & \text { if } \tau_{1}^{*}<\tau<\infty\end{cases}
$$

where $a_{1}$ and $a_{2}$ are two positive constants with $a_{1}>a_{2}$ and $a_{2} / \tau_{2}^{*}=a_{1} / \tau_{1}^{*}$.

The main difficulty is the presence of a constant zone in the pressure law. Indeed, this leads to a local degenerate problem, since for $\tau \in\left[\tau_{2}^{*}, \tau_{1}^{*}\right]$ the eigenvalues $\pm c(\mathbf{u})$ of the system coalesce and the eigenvectors do not form a basis of $\mathbb{R}^{2}$ any longer. Such a problem, usually called resonance, has been widely studied (see for instance $[16,21,22,15,17,29,26,11,6,1])$. In most cases, the resonant region is a hypersurface of the phase space, except in $[25,1]$ (which will also be our case) where the resonant region is a subdomain of the phase space (a point in the scalar case, a curve for a $2 \times 2$ system).

In this work, we study the Riemann problem for (1.1-1.3), i.e. system (1.1-1.3) with the initial condition

$$
\mathbf{u}_{\mid t=0}= \begin{cases}\mathbf{u}_{L} & \text { if } x<0, \\ \mathbf{u}_{R} & \text { if } x>0\end{cases}
$$


where the data $\mathbf{u}_{L}$ and $\mathbf{u}_{R}$ belong to $\Omega$. Such a study is of interest since it is a first step towards the Cauchy problem and it allows the construction of numerical schemes, such as the Godunov scheme [14].

The existence and uniqueness of the solution of the Riemann problem (1.1-1.4) is proved, for any data $\mathbf{u}_{L}, \mathbf{u}_{R} \in \Omega$ (all these results hold similarly for the corresponding system written in Eulerian coordinates). Because of the lack of regularity and the nonconvexity of $\mathscr{P}$, the solution involves multiple waves (as in $[10,30,12])$ and the selection of admissible solutions is performed by the use of the extended entropy condition (E) proposed by Liu [20] (see also [30]). More precisely, the nonconvexity of $\mathscr{P}$ leads to waves which can be composed of rarefaction waves attached with discontinuities (shocks or contact discontinuities) while the loss of regularity of $\mathscr{P}$ at $\tau_{1}^{*}$ and $\tau_{2}^{*}$ introduces constant states inside waves (see for instance Fig.5.3).

The paper is organized as follows. In Section 2, we make precise assumptions about the pressure law $\mathscr{P}$ and state the results of the solution to the Riemann problem (1.1-1.4). A useful geometric interpretation of the entropy condition (E) is presented in Section 3. The Riemann problem is solved by the study of the intersection of two sets of states: the set of nonpositive waves and the set of nonnegative waves. In section 4, we compute the set of nonpositive waves which are involved in the solution of the Riemann problem. In Section 5, the set of nonnegative waves is given and the Riemann problem is solved and shown to be well-posed (existence, uniqueness, $\mathbf{L}_{\text {loc }}^{1}$-continuity).

\section{Assumptions and main results}

We consider a pressure law which complies with the following properties (illustrated by Fig. 2.1). There exists two constant specific volumes $0<\tau_{2}^{*}<\tau_{1}^{*}$ such that:

(H1) $\mathscr{P} \in \mathscr{C}^{0}\left(\mathbb{R}_{+}^{*}, \mathbb{R}_{+}^{*}\right) \cap \mathscr{C}^{2}\left(\left(0, \tau_{2}^{*}\right] \cup\left[\tau_{1}^{*}, \infty\right)\right)$,

(H2) $\mathscr{P}^{\prime}(\tau)<0$ and $\mathscr{P}^{\prime \prime}(\tau)>0$, for all $\tau \in\left(0, \tau_{2}^{*}\right) \cup\left(\tau_{1}^{*}, \infty\right)$,

(H3) $\lim _{\tau \rightarrow 0^{+}} \mathscr{P}(\tau)=+\infty$,

(H4) $\mathscr{P}(\tau)=p_{m}$ for all $\tau \in\left[\tau_{1}^{*}, \tau_{2}^{*}\right]$,

(H5) $\mathscr{P}(\tau)>\mathscr{P}^{\prime}\left(\tau_{2}^{*}\right)\left(\tau-\tau_{2}^{*}\right)+\mathscr{P}\left(\tau_{2}^{*}\right)$, for all $\tau>\tau_{1}^{*}$.

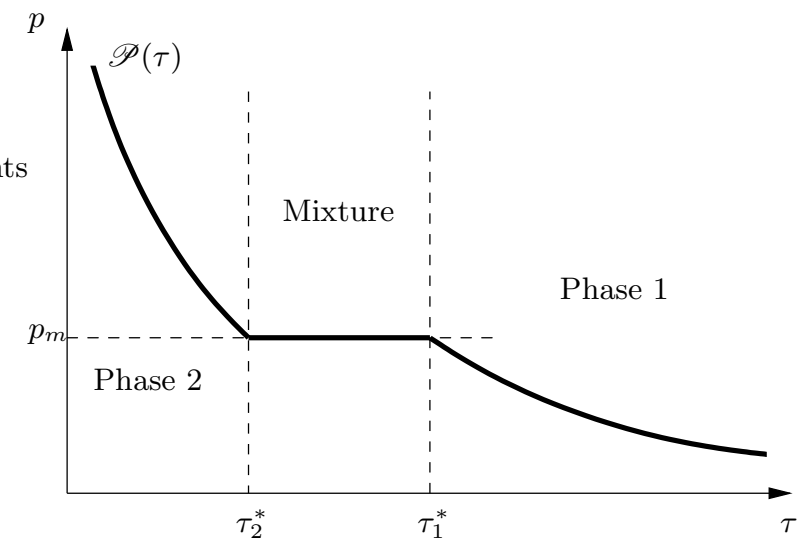

FIG. 2.1. A pressure law fulfilling (H1-5).

The last assumption is not necessary to obtain the results we present but it simplifies the computations. The assumptions on the pressure law in the region of pure phases 
$\left(0, \tau_{2}^{*}\right) \cup\left(\tau_{1}^{*}, \infty\right)$ are described in $(\mathrm{H} 2)$. They can be generalized, following Wendroff [30], but we restrict to (H2) for simplicity.

This pressure law has two non classical features: it is not globally convex and it involves a constant region. This leads to the following behavior according to the region of $\Omega$ we study:

$$
\Omega_{p}=\left(\left(0, \tau_{2}^{*}\right) \cup\left(\tau_{1}^{*}, \infty\right)\right) \times \mathbb{R} \quad \text { and } \quad \Omega_{m}=\left[\tau_{2}^{*}, \tau_{1}^{*}\right] \times \mathbb{R} .
$$

Proposition 2.1. In $\Omega_{p}$, system (1.1-1.3) is strictly hyperbolic: the two eigenvalues

$$
\lambda_{-}(\mathbf{u})=-\sqrt{-\mathscr{P}^{\prime}(\tau)} \quad \text { and } \quad \lambda_{+}(\mathbf{u})=\sqrt{-\mathscr{P}^{\prime}(\tau)}
$$

are real and the corresponding eigenvectors $\mathbf{r}_{-}(\mathbf{u})$ and $\mathbf{r}_{+}(\mathbf{u})$ are linearly independent, for all $\mathbf{u} \in \Omega_{p}$. The two fields are genuinely nonlinear.

In $\Omega_{m}$, system (1.1-1.3) is nonstrictly hyperbolic: $\lambda_{-}(\mathbf{u})=\lambda_{+}(\mathbf{u})=0$ and the corresponding eigenvectors $\mathbf{r}_{-}(\mathbf{u})$ and $\mathbf{r}_{+}(\mathbf{u})$ are linearly dependent, for all $\mathbf{u} \in \Omega_{m}$. The field is linearly degenerate.

The proof of this result is left to the reader.

In the following, the waves associated with the eigenvalue $\lambda_{-}$(respectively $\left.\lambda_{+}\right)$ will be denoted by nonpositive waves (resp. nonnegative waves).

One can remark that for, $\mathbf{u} \in \Omega_{m}$, system (1.1-1.3) becomes the system of pressureless gas $[3,4]$ for which measure solutions need to be introduced. We will see in the sequel that such solutions do not appear because the resonant region $\Omega_{m}$ is bounded with respect to $\tau$.

Let us now recall the entropy condition (E) given by Liu [20]. Let us first define the Rankine-Hugoniot set $\mathscr{S}\left(\mathbf{u}_{l}\right) \subset \Omega \backslash\left\{\mathbf{u}_{l}\right\}$ as the set of $\mathbf{u}_{r} \in \Omega, \mathbf{u}_{l} \neq \mathbf{u}_{r}$, such that there exists $\sigma\left(\mathbf{u}_{l}, \mathbf{u}_{r}\right) \in \mathbb{R}$ with

$$
-\sigma\left(\mathbf{u}_{l}, \mathbf{u}_{r}\right)\left(\mathbf{u}_{r}-\mathbf{u}_{l}\right)+\left(\mathbf{f}\left(\mathbf{u}_{r}\right)-\mathbf{f}\left(\mathbf{u}_{l}\right)\right)=0 .
$$

In the rest of the paper, we use the classical notation $\mathbf{u}_{\alpha}^{\beta}=\left(\tau_{\alpha}^{\beta}, u_{\alpha}^{\beta}\right)$, for any given subscript $\alpha$ and superscript $\beta$.

Definition 2.1. A discontinuity between two states $\mathbf{u}_{l}=\left(\tau_{l}, u_{l}\right) \in \Omega$ and $\mathbf{u}_{r}=$ $\left(\tau_{r}, u_{r}\right) \in \mathscr{S}\left(\mathbf{u}_{l}\right)$ satisfies the entropy condition (E) if

$$
\sigma\left(\mathbf{u}_{l}, \mathbf{u}_{r}\right) \leq \sigma\left(\mathbf{u}_{l}, \mathbf{u}\right)
$$

for all $\mathbf{u} \in \mathscr{S}\left(\mathbf{u}_{l}\right)$ with $\tau \in\left(\min \left(\tau_{l}, \tau_{r}\right), \max \left(\tau_{l}, \tau_{r}\right)\right)$. A discontinuity which satisfies the entropy condition (E) will be called an admissible discontinuity.

When considering the Riemann problem (1.1-1.4), we look for solutions in the class of self-similar solutions composed by a succession of rarefaction waves, admissible discontinuities and constant states. Two cases must be distinguished:

$$
\begin{aligned}
& \text { (H6a) } \int_{\tau_{1}^{*}}^{+\infty} \sqrt{-\mathscr{P}^{\prime}(\tau)} d \tau=+\infty, \\
& \text { (H6b) } \int_{\tau_{1}^{*}}^{+\infty} \sqrt{-\mathscr{P}^{\prime}(\tau)} d \tau<+\infty .
\end{aligned}
$$

In the first case, the vacuum never appears, whereas in the second case, the Riemann problem may have no solution if one does not introduce the vacuum $[23,28,13]$. We have chosen to introduce the vacuum, that is $\{\tau=+\infty, u \in \mathbb{R}\}$, in order to simplify 
the presentation, all the more since explicit conditions of occurrence of the vacuum are difficult to obtain.

We can now state the following result:

TheOREM 2.2. For all $\mathbf{u}_{L}, \mathbf{u}_{R} \in \Omega$, the solution of the Riemann problem (1.1-1.4):

i. exists and is unique if (H6a) is fulfilled,

ii. exists and is unique if (H6b) is fulfilled, allowing the occurrence of the vacuum state $\{\tau=+\infty\}$ when necessary.

Restricting to data in $\Omega_{p}$, Colombo and Priuli [7] have already proved such a result (and extended it to the use of kinetic relations). Besides, the solution satisfies the following comparison result:

THEOREM 2.3. Let $\mathscr{W}\left(x / t ; \mathbf{u}_{L}, \mathbf{u}_{R}\right)$ be the self-similar solution of the Riemann problem (1.1-1.4). For any $L>0$ and initial data $\left(\mathbf{u}_{L}, \mathbf{u}_{R}\right),\left(\mathbf{v}_{L}, \mathbf{v}_{R}\right) \in \Omega^{2}$, there exists a constant $C>0$ such that

$$
\int_{-L}^{L}\left|\mathscr{W}\left(\xi ; \mathbf{u}_{L}, \mathbf{u}_{R}\right)-\mathscr{W}\left(\xi ; \mathbf{v}_{L}, \mathbf{v}_{R}\right)\right| d \xi \leq C\left(\left|\mathbf{u}_{L}-\mathbf{v}_{L}\right|+\left|\mathbf{u}_{R}-\mathbf{v}_{R}\right|\right)
$$

where $|\cdot|$ stands for the Euclidean norm of $\mathbb{R}^{2}$.

The proof of Thm. 2.2 follows three steps. The first one consists in a rewriting of the condition (E), initially proposed by Wendroff in [30]. Secondly, we investigate all the states which can be connected to a given state by a succession of nonpositive waves (respectively nonnegative waves), possibly separated by constant states, that is to say which correspond to the eigenvalue $\lambda_{-}(\mathbf{u})$ (resp. $\left.\lambda_{+}(\mathbf{u})\right)$ which is nonpositive (resp. nonnegative)). Finally, the solution of the Riemann problem is studied. Existence is obtained with the use of the vacuum state while uniqueness is achieved using the property of self-similarity of the solution. Theorem 2.3 is a direct consequence of the structure of the solution.

\section{A geometric interpretation of entropy condition (E)}

We recall here a classical result, which can be found in [30] (see also [8, 9, 18] for other entropy criteria and their equivalence).

LEMma 3.1. Let $\mathbf{u}_{l}$ be in $\Omega$ and $\mathbf{u}_{r} \in \mathscr{S}\left(\mathbf{u}_{l}\right)$, with $\tau_{l} \neq \tau_{r}$.

Then, $\mathbf{u}_{l}$ and $\mathbf{u}_{r}$ are separated by an admissible discontinuity (i.e. satisfying (2.3)) if and only if

$$
\sigma\left(\mathbf{u}_{l}, \mathbf{u}_{r}\right)\left(\frac{\mathscr{P}\left(\tau_{r}\right)-\mathscr{P}\left(\tau_{l}\right)}{\tau_{r}-\tau_{l}}-\frac{\mathscr{P}(\tau)-\mathscr{P}\left(\tau_{l}\right)}{\tau-\tau_{l}}\right) \geq 0
$$

for all $\tau \in\left(\min \left(\tau_{l}, \tau_{r}\right), \max \left(\tau_{l}, \tau_{r}\right)\right)$.

Proof. By definition of the Rankine-Hugoniot set $\mathscr{S}\left(\mathbf{u}_{l}\right)$, we have

$$
\begin{array}{r}
-\sigma\left(\mathbf{u}_{l}, \mathbf{u}_{r}\right)\left(\tau_{r}-\tau_{l}\right)-\left(u_{r}-u_{l}\right)=0 \\
-\sigma\left(\mathbf{u}_{l}, \mathbf{u}_{r}\right)\left(u_{r}-u_{l}\right)+\left(\mathscr{P}\left(\tau_{r}\right)-\mathscr{P}\left(\tau_{l}\right)\right)=0 .
\end{array}
$$

Combining both equations yields

$$
\sigma\left(\mathbf{u}_{l}, \mathbf{u}_{r}\right)=-\frac{u_{r}-u_{l}}{\tau_{r}-\tau_{l}}
$$


and

$$
\left(u_{r}-u_{l}\right)^{2}=-\left(\mathscr{P}\left(\tau_{r}\right)-\mathscr{P}\left(\tau_{l}\right)\right)\left(\tau_{r}-\tau_{l}\right) .
$$

Therefore, we obtain

$$
\left(\sigma\left(\mathbf{u}_{l}, \mathbf{u}_{r}\right)\right)^{2}=-\frac{\mathscr{P}\left(\tau_{r}\right)-\mathscr{P}\left(\tau_{l}\right)}{\tau_{r}-\tau_{l}} .
$$

The same calculations hold for $\mathbf{u}_{l}$ and $\mathbf{u} \in \mathscr{S}\left(\mathbf{u}_{l}\right)$. Then, if $\sigma\left(\mathbf{u}_{l}, \mathbf{u}_{r}\right) \geq 0$, inequality (2.3) becomes

$$
\frac{\mathscr{P}\left(\tau_{r}\right)-\mathscr{P}\left(\tau_{l}\right)}{\tau_{r}-\tau_{l}} \geq \frac{\mathscr{P}(\tau)-\mathscr{P}\left(\tau_{l}\right)}{\tau-\tau_{l}} .
$$

The result for the case $\sigma\left(\mathbf{u}_{l}, \mathbf{u}_{r}\right) \leq 0$ follows similarly.

REMARK 3.2. This result can easily be interpreted geometrically. Assume that the speed $\sigma\left(\mathbf{u}_{l}, \mathbf{u}_{r}\right)$ of the discontinuity between $\mathbf{u}_{l}$ and $\mathbf{u}_{r}$ is nonpositive, that is to say that we consider a nonpositive wave $\lambda_{-}$. If $\tau_{l}<\tau_{r}$, then in the $(\tau, p)$ plane, the segment linking $\left(\tau_{l}, \mathscr{P}\left(\tau_{l}\right)\right)$ to $\left(\tau_{r}, \mathscr{P}\left(\tau_{r}\right)\right)$ must be below the curve $\left\{(\tau, p) \in\left(\tau_{l}, \tau_{r}\right) \times \mathbb{R}_{+}, p=\right.$ $\mathscr{P}(\tau)\}$ (in the non-strict sense) or in other words, the graph of the function $\mathscr{P}$ lies above the chord. Similarly, if $\tau_{l}>\tau_{r}$, the segment $\left[\left(\tau_{l}, \mathscr{P}\left(\tau_{l}\right)\right),\left(\tau_{r}, \mathscr{P}\left(\tau_{r}\right)\right)\right]$ must be above the curve $\left\{(\tau, p) \in\left(\tau_{r}, \tau_{l}\right) \times \mathbb{R}_{+}, p=\mathscr{P}(\tau)\right\}$ (see also Remark 4.4). Of course, a similar geometric interpretation holds for nonnegative waves $\lambda_{+}$too.

Note that this characterization is similar to the one in the scalar case with nonconvex flux functions [12].

We focus now on the characterization of nonpositive waves. Due to the nonconvexity of the pressure law, such a wave can be a succession of rarefaction waves and admissible discontinuities, all corresponding to the eigenvalue $\lambda_{-}$.

\section{Nonpositive multiple waves}

In this section, we seek all the states which can be connected to a given state, say $\mathbf{u}_{L}$, by nonpositive waves. First, we focus on single rarefaction waves and single admissible discontinuities associated with the eigenvalue $\lambda_{-}$. Afterwards, we construct nonpositive multiple waves as successions of these single waves.

4.1. Nonpositive rarefaction waves. Using Prop. 2.1, the study of rarefaction waves can be restricted to $\Omega_{p}$. We note $\mathscr{R}_{-}\left(\mathbf{u}_{L}\right)$ the set of the states which can be connected to $\mathbf{u}_{L}$ by a nonpositive rarefaction wave. If we define the function $\phi_{-}$ by

$$
\begin{aligned}
\phi_{-}: \mathbb{R}_{+}^{*} \times \Omega & \longmapsto \mathbb{R} \\
\left(\tau, \mathbf{u}_{L}\right) & \longmapsto u_{L}+\int_{\tau_{L}}^{\tau} \sqrt{-\mathscr{P}^{\prime}(v)} d v
\end{aligned}
$$

the set $\mathscr{R}_{-}\left(\mathbf{u}_{L}\right)$ can be explicitly stated $[28,13]$ :

Lemma 4.1. The set $\mathscr{R}_{-}\left(\mathbf{u}_{L}\right)$ of the states which can be connected to $\mathbf{u}_{L}$ by a nonpositive rarefaction wave is given by:

- If $\tau_{L}<\tau_{2}^{*}, \mathscr{R}_{-}\left(\mathbf{u}_{L}\right)=\left\{\left(\tau, \phi_{-}\left(\tau, \mathbf{u}_{L}\right)\right), \tau \in\left(\tau_{L}, \tau_{2}^{*}\right]\right\}$.

- If $\tau_{L}>\tau_{1}^{*}, \mathscr{R}_{-}\left(\mathbf{u}_{L}\right)=\left\{\left(\tau, \phi_{-}\left(\tau, \mathbf{u}_{L}\right)\right), \tau \in\left(\tau_{L}, \infty\right)\right\}$. 
Both curves are increasing and concave in the $(\tau, u)$ plane.

Furthermore, if hypothesis (H6a) holds and $\tau_{L}>\tau_{1}^{*}$, the curve $\mathscr{R}_{-}\left(\mathbf{u}_{L}\right)$ is unbounded by the above in the $(\tau, u)$ plane, with respect to $u$. However, if hypothesis (H6b) is fulfilled and $\tau_{L}>\tau_{1}^{*}$, then the curve $\mathscr{R}_{-}\left(\mathbf{u}_{L}\right)$ admits a horizontal asymptote, namely $\mathbb{R}_{+}^{*} \times\left\{u_{L}+u_{\infty}\left(\tau_{L}\right)\right\}$, where

$$
u_{\infty}\left(\tau_{L}\right)=\int_{\tau_{L}}^{\infty} \sqrt{-\mathscr{P}^{\prime}(v)} d v<\infty .
$$

This classical behavior is crucial when dealing with global initial data.

4.2. Nonpositive admissible discontinuities. We focus now on admissible discontinuities. Let $\mathscr{S}_{-}\left(\mathbf{u}_{L}\right)$ be the set of states which can be connected to $\mathbf{u}_{L}$ by an admissible discontinuity (in the sense of Def. 2.1) with a nonpositive speed. In order to describe this set, let us first introduce some notations.

We define $\tau_{\sharp}$ as the function which maps any $\tau_{L} \in\left[\tau_{2}^{*}, \tau_{1}^{*}\right)$ to the unique element of

$$
\left\{\tau>\tau_{1}^{*}, \mathscr{P}(\tau)-\mathscr{P}\left(\tau_{L}\right)=\mathscr{P}^{\prime}(\tau)\left(\tau-\tau_{L}\right)\right\}
$$

In the same way, we note $\tau_{b}$ as the function which maps any $\tau_{L} \in\left(\tau_{1}^{*}, \infty\right)$ to the unique element of

$$
\left\{\tau<\tau_{2}^{*},\left(\mathscr{P}(\tau)-\mathscr{P}\left(\tau_{L}\right)\right)\left(\tau_{1}^{*}-\tau_{L}\right)=\left(\mathscr{P}\left(\tau_{1}^{*}\right)-\mathscr{P}\left(\tau_{L}\right)\right)\left(\tau-\tau_{L}\right)\right\}
$$

These two functions are monovalued, thanks to the assumptions on the pressure law $\mathscr{P}$. An illustration of these functions is given in Fig. 4.1. Now, if we define the function

$$
\begin{aligned}
\psi_{-}: \mathbb{R}_{+}^{*} \times \Omega & \longmapsto \mathbb{R} \\
\left(\tau, \mathbf{u}_{L}\right) & \longmapsto u_{L}+\operatorname{sign}\left(\tau-\tau_{L}\right) \sqrt{\left(\mathscr{P}(\tau)-\mathscr{P}\left(\tau_{L}\right)\right)\left(\tau_{L}-\tau\right)},
\end{aligned}
$$

we are in position to define more precisely the set of nonpositive admissible discontinuities $\mathscr{S}_{-}\left(\mathbf{u}_{L}\right)$ :

LEMma 4.2. The set $\mathscr{S}_{-}\left(\mathbf{u}_{L}\right)$ of the states which can be reached from $\mathbf{u}_{L}$ by a nonpositive admissible discontinuity is given by:

- If $\tau_{L} \in\left(0, \tau_{2}^{*}\right), \mathscr{S}_{-}\left(\mathbf{u}_{L}\right)=\left\{\left(\tau, \psi_{-}\left(\tau, \mathbf{u}_{L}\right)\right), \tau \in\left(0, \tau_{L}\right)\right\}$.

- If $\tau_{L} \in\left[\tau_{2}^{*}, \tau_{1}^{*}\right], \mathscr{S}_{-}\left(\mathbf{u}_{L}\right)=\left\{\left(\tau, \psi_{-}\left(\tau, \mathbf{u}_{L}\right)\right), \tau \in\left(0, \tau_{\sharp}\left(\tau_{L}\right)\right]\right\}$.

- If $\tau_{L} \in\left(\tau_{1}^{*}, \infty\right), \mathscr{S}_{-}\left(\mathbf{u}_{L}\right)=\left\{\left(\tau, \psi_{-}\left(\tau, \mathbf{u}_{L}\right)\right), \tau \in\left(0, \tau_{b}\left(\tau_{L}\right)\right] \cup\left[\tau_{1}^{*}, \tau_{L}\right)\right\}$.

Moreover, the function $\psi_{-}$verifies for all $\mathbf{u}_{L} \in \Omega$

- $\psi_{-}\left(\cdot, \mathbf{u}_{L}\right) \in \mathscr{C}^{0}\left(\mathbb{R}_{+}^{*}\right) \cap \mathscr{C}^{2}\left(\left(0, \tau_{2}^{*}\right] \cup\left[\tau_{1}^{*}, \infty\right)\right)$.

- $\partial_{\tau} \psi_{-}\left(\tau, \mathbf{u}_{L}\right)>0$ and $\partial_{\tau \tau}^{2} \psi_{-}\left(\tau, \mathbf{u}_{L}\right)<0$, for all $\tau \in\left(0, \tau_{2}^{*}\right) \cup\left(\tau_{1}^{*}, \infty\right)$.

- $\lim _{\tau \rightarrow 0^{+}} \psi_{-}\left(\tau, \mathbf{u}_{L}\right)=-\infty$.

- $\psi_{-}\left(\tau, \mathbf{u}_{L}\right)=\psi_{-}\left(\tau_{2}^{*}, \mathbf{u}_{L}\right)=\psi_{-}\left(\tau_{1}^{*}, \mathbf{u}_{L}\right)$ for all $\tau \in\left[\tau_{1}^{*}, \tau_{2}^{*}\right]$.

Proof. The characterization of the set $\mathscr{S}_{-}\left(\mathbf{u}_{L}\right)$ is directly given by Lem. 3.1 and Rem. 3.2 and the properties of the function $\psi_{-}$are based on classical calculations, using hypotheses $(\mathrm{H} 1-5)$ made on the pressure law.

The set $\mathscr{S}_{-}\left(\mathbf{u}_{L}\right)$ is represented in the $(\tau, p)$ plane by bold lines on Fig. 4.1. 

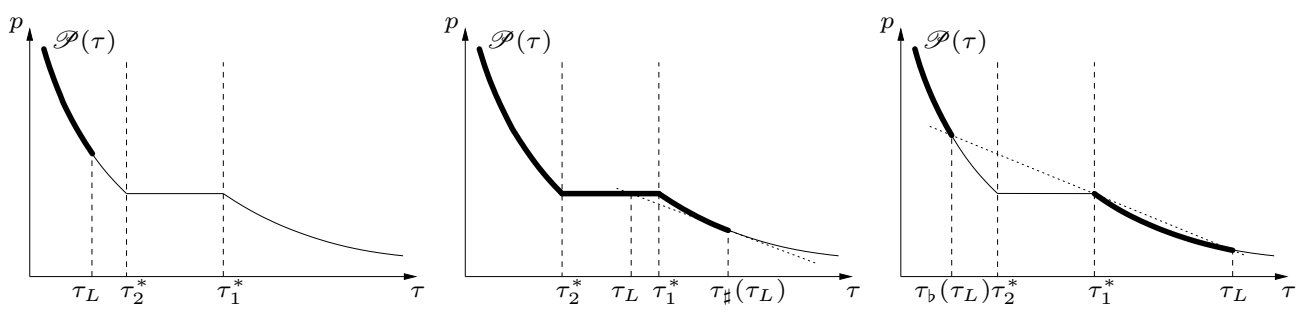

$0<\tau_{L}<\tau_{2}^{*}$

$$
\tau_{2}^{*}<\tau_{L}<\tau_{1}^{*}
$$$$
\tau_{1}^{*}<\tau_{L}
$$

FIG. 4.1. Admissible discontinuities (bold parts of the curves).

In Fig. 4.2 are shown the sets $\left.\mathscr{S}_{-}\left(\mathbf{u}_{L}\right)\right)$ and $\mathscr{R}_{-}\left(\mathbf{u}_{L}\right)$, with respect to the location of $\tau_{L}$ in the $(\tau, u)$ plane. One may note that

$$
\lim _{\tau_{L} \geq \tau_{1}^{*}} \mathscr{S}_{-}\left(\mathbf{u}_{L}\right) \subsetneq \lim _{\tau_{L} \leq \tau_{1}^{*}} \mathscr{S}_{-}\left(\mathbf{u}_{L}\right)
$$

since $\tau_{b}\left(\tau_{L}\right)$ is not defined when $\tau_{L}=\tau_{1}^{*}$. This is due to the fact that $\mathscr{P}$ is only Lipschitz continuous at $\tau_{1}^{*}$, but it does not alter the global resolution of the Riemann problem since we use multiple waves (see Section 4.3 .3 where the set of nonpositive multiple waves is shown to be continuous).
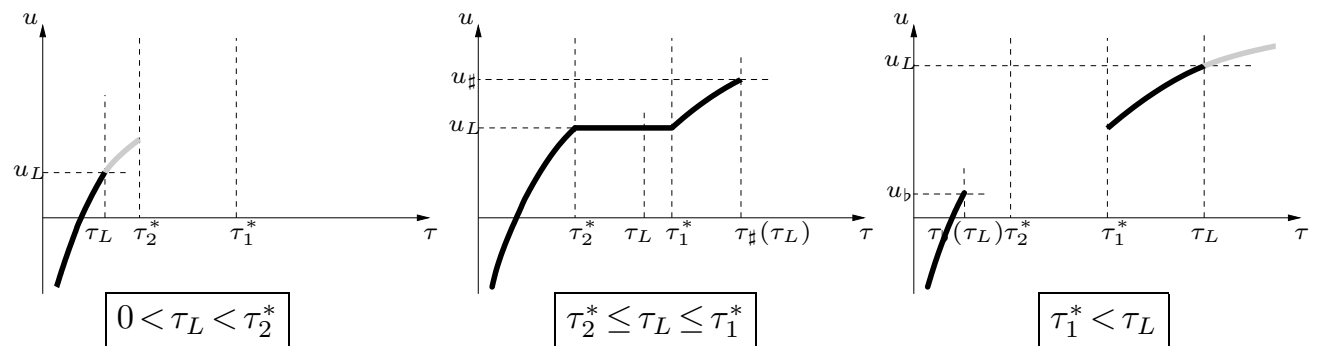

FIG. 4.2. Curves $\mathscr{R}_{-}\left(\mathbf{u}_{L}\right)$ (grey line) and $\mathscr{S}_{-}\left(\mathbf{u}_{L}\right)$ (black line).

4.3. Construction of nonpositive multiple waves. We have constructed the set of states which can be reached from $\mathbf{u}_{L}$ by one nonpositive wave: $\mathscr{R}_{-}\left(\mathbf{u}_{L}\right) \cup$ $\mathscr{S}_{-}\left(\mathbf{u}_{L}\right)$. However, this set is incomplete (see Fig. 4.2). It is thus necessary to consider multiple waves. Such waves are classical when dealing with nonconvex pressure laws $[10,30,20]$.

First, let us remark that, since the flux $\mathbf{f}$ is independent of $t$ and $x$, the solution of the Riemann problem is self-similar. Then, a nonpositive multiple wave is simply a succession of nonpositive rarefaction waves, admissible discontinuities and constant states. If $\mathscr{U}_{-}\left(\mathbf{u}_{L}\right)$ denotes the set of states which can be connected to $\mathbf{u}_{L}$ by a nonpositive multiple wave, that is to say

$$
\mathscr{U}_{-}\left(\mathbf{u}_{L}\right)=\left\{\mathscr{W}\left(0^{+} ; \mathbf{u}_{L}, \mathbf{u}\right), \mathbf{u} \in \Omega\right\}
$$

where $\mathscr{W}\left(x / t ; \mathbf{u}_{L}, \mathbf{u}_{R}\right)$ is a self-similar solution of the Riemann problem (1.1-1.4), $\mathscr{U}_{-}\left(\mathbf{u}_{L}\right)$ will at least contain the set $\left\{\mathbf{u}_{L}\right\} \cup \mathscr{R}_{-}\left(\mathbf{u}_{L}\right) \cup \mathscr{S}_{-}\left(\mathbf{u}_{L}\right)$. In order to construct multiple waves, we will introduce intermediate states (to be determined), say $\mathbf{u}_{i}$, and 
consider $\mathscr{R}_{-}\left(\mathbf{u}_{i}\right)$ and $\mathscr{S}_{-}\left(\mathbf{u}_{i}\right)$ in such a way that the speeds of consecutive waves are in increasing order.

The study of $\mathscr{U}_{-}\left(\mathbf{u}_{L}\right)$ is split into three parts, following the location of $\mathbf{u}_{L}$ : in the mixture zone $\left(\tau_{2}^{*} \leq \tau_{L} \leq \tau_{1}^{*}\right)$, in phase $2\left(0<\tau_{L}<\tau_{2}^{*}\right)$ and in phase $1\left(\tau_{1}^{*}<\tau_{L}\right)$.

4.3.1. The left state is in the mixture zone. Surprisingly, this case is the simplest one. In this case, the only state from which we can restart is

$$
\mathbf{u}_{\sharp}\left(\mathbf{u}_{L}\right)=\left(\tau_{\sharp}\left(\tau_{L}\right), \psi_{-}\left(\tau_{\sharp}\left(\tau_{L}\right), \mathbf{u}_{L}\right)\right)
$$

(see Lem. 4.2 and Fig. 4.2-center). It remains to compare the speed of the discontinuity which links $\mathbf{u}_{L}$ and $\mathbf{u}_{\sharp}\left(\mathbf{u}_{L}\right)$ with the speed of the wave which links $\mathbf{u}_{\sharp}\left(\mathbf{u}_{L}\right)$ with any state of $\mathscr{R}_{-}\left(\mathbf{u}_{\sharp}\left(\mathbf{u}_{L}\right)\right) \cup \mathscr{S}_{-}\left(\mathbf{u}_{\sharp}\left(\mathbf{u}_{L}\right)\right)$.

Due to the definition of $\tau_{\sharp}\left(\tau_{L}\right)$, the speed of the discontinuity between $\mathbf{u}_{L}$ and $\mathbf{u}_{\sharp}\left(\mathbf{u}_{L}\right)$ is exactly

$$
\sigma\left(\mathbf{u}_{L}, \mathbf{u}_{\sharp}\left(\mathbf{u}_{L}\right)\right)=-\sqrt{-\mathscr{P}^{\prime}\left(\tau_{\sharp}\left(\tau_{L}\right)\right)} .
$$

It is easy to check that for any state $\mathbf{u} \in \mathscr{S}_{-}\left(\mathbf{u}_{\sharp}\left(\mathbf{u}_{L}\right)\right)$,

$$
\sigma\left(\mathbf{u}_{\sharp}\left(\mathbf{u}_{L}\right), \mathbf{u}\right)<\sigma\left(\mathbf{u}_{L}, \mathbf{u}_{\sharp}\left(\mathbf{u}_{L}\right)\right) .
$$

Therefore, $\mathscr{U}_{-}\left(\mathbf{u}_{L}\right) \cap \mathscr{S}_{-}\left(\mathbf{u}_{\sharp}\left(\mathbf{u}_{L}\right)\right)=\emptyset$. Consider now the set $\mathscr{R}_{-}\left(\mathbf{u}_{\sharp}\left(\mathbf{u}_{L}\right)\right)$. For any $\mathbf{u} \in \mathscr{R}_{-}\left(\mathbf{u}_{\sharp}\left(\mathbf{u}_{L}\right)\right)$, the speed $\lambda_{-}(\mathbf{u})=-\sqrt{-\mathscr{P}^{\prime}(\tau)}$ is increasing, then $\mathscr{R}_{-}\left(\mathbf{u}_{\sharp}\left(\mathbf{u}_{L}\right)\right) \subset$ $\mathscr{U}_{-}\left(\mathbf{u}_{L}\right)$. Finally, we can define

$$
\mathscr{U}_{-}\left(\mathbf{u}_{L}\right)=\left\{\mathbf{u}_{L}\right\} \cup \mathscr{S}_{-}\left(\mathbf{u}_{L}\right) \cup \mathscr{R}_{-}\left(\mathbf{u}_{\sharp}\left(\mathbf{u}_{L}\right)\right) .
$$

Let us note that, by Eq. (4.5), the discontinuity between $\mathbf{u}_{L}$ and $\mathbf{u}_{\sharp}\left(\mathbf{u}_{L}\right)$ and the rarefaction wave starting from $\mathbf{u}_{\sharp}\left(\mathbf{u}_{L}\right)$ are attached (see Fig. 4.3-bottom).

4.3.2. The left state is in phase 2. We consider now waves starting from the state

$$
\mathbf{u}_{2}^{*}\left(\mathbf{u}_{L}\right)=\left(\tau_{2}^{*}, \phi_{-}\left(\tau_{2}^{*}, \mathbf{u}_{L}\right)\right)
$$

which corresponds to the second case of Lem. 4.2 and Fig. 4.2-left. Any discontinuity between $\mathbf{u}_{2}^{*}\left(\mathbf{u}_{L}\right)$ and a state $\mathbf{u} \in \mathscr{S}_{-}\left(\mathbf{u}_{2}^{*}\left(\mathbf{u}_{L}\right)\right)$ satisfying $\tau<\tau_{2}^{*}$ moves with a speed $\sigma\left(\mathbf{u}_{2}^{*}\left(\mathbf{u}_{L}\right), \mathbf{u}\right)$ which is smaller than $-\sqrt{-\mathscr{P}^{\prime}\left(\tau_{2}^{*}-0\right)}$ (the speed of the tail of the rarefaction wave between $\mathbf{u}_{L}$ and $\left.\mathbf{u}_{2}^{*}\left(\mathbf{u}_{L}\right)\right)$. Thus, no state of $\mathscr{S}_{-}\left(\mathbf{u}_{2}^{*}\left(\mathbf{u}_{L}\right)\right) \cap\left(\left(0, \tau_{2}^{*}\right) \times\right.$ $\mathbb{R})$ belongs to $\mathscr{U}_{-}\left(\mathbf{u}_{L}\right)$.

On the contrary, all the remaining parts of $\mathscr{S}_{-}\left(\mathbf{u}_{2}^{*}\left(\mathbf{u}_{L}\right)\right.$ ) (that is for specific volumes between $\tau_{2}^{*}$ and $\left.\tau_{\sharp}\left(\tau_{2}^{*}\right)\right)$ are included in $\mathscr{U}_{-}\left(\mathbf{u}_{L}\right)$. This is a direct consequence of hypothesis $(\mathrm{H} 5)$ : the speed of the end of the rarefaction wave between $\mathbf{u}_{L}$ and $\mathbf{u}_{2}^{*}\left(\mathbf{u}_{L}\right)$ is smaller than the speed of the discontinuity between $\mathbf{u}_{2}^{*}\left(\mathbf{u}_{L}\right)$ and any state of $\mathscr{S}_{-}\left(\mathbf{u}_{2}^{*}\left(\mathbf{u}_{L}\right)\right) \cap\left(\left(\tau_{2}^{*}, \tau_{\sharp}\left(\tau_{2}^{*}\right)\right) \times \mathbb{R}\right)$. Since this inequality is strict, the rarefaction wave and the discontinuity are not attached but separated by the state $\mathbf{u}_{2}^{*}\left(\mathbf{u}_{L}\right)$.

The set $\mathscr{U}_{-}\left(\mathbf{u}_{L}\right)$ is not complete yet since $\mathscr{S}_{-}\left(\mathbf{u}_{2}^{*}\left(\mathbf{u}_{L}\right)\right)$ stops at the state

$$
\mathbf{u}_{\sharp}^{*}\left(\mathbf{u}_{L}\right)=\left(\tau_{\sharp}\left(\tau_{2}^{*}\right), \psi_{-}\left(\tau_{\sharp}\left(\tau_{2}^{*}\right), \mathbf{u}_{2}^{*}\left(\mathbf{u}_{L}\right)\right)\right) .
$$

Such a configuration has already been studied in the previous case: all the states which can be reached by $\mathbf{u}_{\sharp}^{*}\left(\mathbf{u}_{L}\right)$ are given by $\mathscr{R}_{-}\left(\mathbf{u}_{\sharp}^{*}\left(\mathbf{u}_{L}\right)\right)$. Thus, we obtain

$$
\begin{gathered}
\mathscr{U}_{-}\left(\mathbf{u}_{L}\right)=\left\{\mathbf{u}_{L}\right\} \cup \mathscr{R}_{-}\left(\mathbf{u}_{L}\right) \cup \mathscr{S}_{-}\left(\mathbf{u}_{L}\right) \\
\cup\left(\mathscr{S}_{-}\left(\mathbf{u}_{2}^{*}\left(\mathbf{u}_{L}\right)\right) \cap\left(\left[\tau_{2}^{*}, \tau_{\sharp}\left(\tau_{2}^{*}\right)\right] \times \mathbb{R}\right)\right) \cup \mathscr{R}_{-}\left(\mathbf{u}_{\sharp}^{*}\left(\mathbf{u}_{L}\right)\right) .
\end{gathered}
$$


In order to describe more explicitly the waves which can occur in this case, let us take a state $\mathbf{u}=(\tau, u) \in \mathscr{U}_{-}\left(\mathbf{u}_{L}\right)$ :

- If $0<\tau<\tau_{L}$, the states $\mathbf{u}_{L}$ and $\mathbf{u}$ are separated by an admissible discontinuity (corresponding to the black solid line in Fig. 4.3-left).

- If $\tau_{L}<\tau<\tau_{2}^{*}$, the states $\mathbf{u}_{L}$ and $\mathbf{u}$ are separated by a rarefaction wave (corresponding to the grey solid line in Fig. 4.3-left).

- If $\tau_{2}^{*}<\tau<\tau_{\sharp}\left(\tau_{1}^{*}\right)$, the states $\mathbf{u}_{L}$ and $\mathbf{u}$ are separated by a rarefaction wave connecting $\mathbf{u}_{L}$ to $\mathbf{u}_{2}^{*}\left(\mathbf{u}_{L}\right)$ and an admissible discontinuity from $\mathbf{u}_{2}^{*}\left(\mathbf{u}_{L}\right)$ to $\mathbf{u}$ (corresponding to the black dashed line in Fig. 4.3-left).

- If $\tau>\tau_{\sharp}\left(\tau_{1}^{*}\right)$, the states $\mathbf{u}_{L}$ and $\mathbf{u}$ are separated by a rarefaction wave, the state $\mathbf{u}_{2}^{*}\left(\mathbf{u}_{L}\right)$, an admissible discontinuity to which a rarefaction wave is attached at the point $\mathbf{u}_{\sharp}^{*}\left(\mathbf{u}_{L}\right)$ (corresponding to the grey dashed line in Fig. 4.3-left).

4.3.3. The left state is in phase 1 . We are now interested in the state

$$
\mathbf{u}_{1}^{*}\left(\mathbf{u}_{L}\right)=\left(\tau_{1}^{*}, \psi_{-}\left(\tau_{1}^{*}, \mathbf{u}_{L}\right)\right)
$$

which is connected to $\mathbf{u}_{L}$ by a discontinuity. This state fits the second case of Lem. 4.2 and Fig. 4.2-right. Consider now a state $\mathbf{u} \in \mathscr{S}_{-}\left(\mathbf{u}_{1}^{*}\right)$. If its specific volume $\tau$ is greater than $\tau_{1}^{*}$, it must be removed. If $\tau \in\left(\tau_{b}\left(\tau_{L}\right), \tau_{1}^{*}\right)$, one can easily check that $\sigma\left(\mathbf{u}_{1}^{*}, \mathbf{u}\right)>\sigma\left(\mathbf{u}_{L}, \mathbf{u}_{1}^{*}\right)$, that is to say the discontinuities between $\mathbf{u}_{1}^{*}$ and $\mathbf{u}$ and between $\mathbf{u}_{L}$ and $\mathbf{u}_{1}^{*}$ have compatible speeds. If $\tau=\tau_{b}\left(\tau_{L}\right)$, the two discontinuities have the same speed and actually are joined (this is the main argument for the continuity of $\mathscr{U}_{-}\left(\mathbf{u}_{L}\right)$ when $\tau_{L}$ crosses $\left.\tau_{1}^{*}\right)$.

Let us focus now on the states $\mathbf{u}$ with $\tau<\tau_{b}\left(\tau_{L}\right)$. A priori, they can be connected to $\mathbf{u}_{L}$ by two ways. The first one uses the curve $\mathscr{S}_{-}\left(\mathbf{u}_{L}\right)$, linking $\mathbf{u}_{L}$ and $\mathbf{u}$ by a simple discontinuity. The second one uses $\mathscr{S}_{-}\left(\mathbf{u}_{1}^{*}\right)$, linking $\mathbf{u}_{L}$ and $\mathbf{u}$ through two discontinuities separated by the state $\mathbf{u}_{1}^{*}$. But in the second case, the discontinuities are incompatible since $\sigma\left(\mathbf{u}_{1}^{*}, \mathbf{u}\right)<\sigma\left(\mathbf{u}_{L}, \mathbf{u}_{1}^{*}\right)$. We can then define

$$
\begin{aligned}
\mathscr{U}_{-}\left(\mathbf{u}_{L}\right)= & \left\{\mathbf{u}_{L}\right\} \cup \mathscr{R}_{-}\left(\mathbf{u}_{L}\right) \cup \mathscr{S}_{-}\left(\mathbf{u}_{L}\right) \\
& \cup\left(\mathscr{S}_{-}\left(\mathbf{u}_{1}^{*}\left(\mathbf{u}_{L}\right)\right) \cap\left(\left[\tau_{b}\left(\tau_{L}\right), \tau_{1}^{*}\right] \times \mathbb{R}\right)\right) .
\end{aligned}
$$

Note that, by definition of $\tau_{b}\left(\tau_{L}\right)$,

$$
\psi_{-}\left(\tau_{b}\left(\tau_{L}\right), \mathbf{u}_{L}\right)=\psi_{-}\left(\tau_{b}\left(\tau_{L}\right), \mathbf{u}_{1}^{*}\left(\mathbf{u}_{L}\right)\right)
$$

Therefore, the curve $\mathscr{U}_{-}\left(\mathbf{u}_{L}\right)$ is continuous (in the two previous cases, the continuity of $\mathscr{U}_{-}\left(\mathbf{u}_{L}\right)$ was trivial). Moreover, the transition when $\tau_{L}$ crosses $\tau_{1}^{*}$ is continuous too.

As before, let us choose a state $\mathbf{u}$ in $\mathscr{U}_{-}\left(\mathbf{u}_{L}\right)$ and describe the waves which occur, according to the value of $\tau$ :

- If $\tau>\tau_{L}$, the states $\mathbf{u}_{L}$ and $\mathbf{u}$ are separated by a rarefaction wave (corresponding to the grey solid line in Fig. 4.3-right).

- If $\tau_{1}^{*}<\tau<\tau_{L}$ or $\tau<\tau_{b}\left(\tau_{L}\right)$, the states $\mathbf{u}_{L}$ and $\mathbf{u}$ are separated by an admissible discontinuity (corresponding to the black solid lines in Fig. 4.3-right).

- If $\tau_{b}\left(\tau_{L}\right)<\tau<\tau_{1}^{*}$, the states $\mathbf{u}_{L}$ and $\mathbf{u}$ are separated by an admissible discontinuity connecting $\mathbf{u}_{L}$ to $\mathbf{u}_{1}^{*}\left(\mathbf{u}_{L}\right)$ and another admissible discontinuity from $\mathbf{u}_{1}^{*}\left(\mathbf{u}_{L}\right)$ to $\mathbf{u}$ (corresponding to the black dashed line in Fig. 4.3-right). 
4.3.4. The complete parametrization of nonpositive waves. As a result of the previous calculations, whatever the state $\mathbf{u}_{L}$, the curve $\mathscr{U}_{-}\left(\mathbf{u}_{L}\right)$ can be parametrized with respect to $\tau$ and we denote by $\vartheta_{-}: \mathbb{R}_{+}^{*} \times \Omega \longmapsto \mathbb{R}$ the parametrization we have obtained, i.e.

$$
\left\{\left(\tau, \vartheta_{-}\left(\tau, \mathbf{u}_{L}\right)\right), \tau>0\right\}=\mathscr{U}_{-}\left(\mathbf{u}_{L}\right)
$$

The properties of this function can be easily described:

Proposition 4.3. The function $\vartheta_{-}$satisfies for all $\mathbf{u}_{L} \in \Omega$

i. $\vartheta_{-} \in \mathscr{C}^{0}\left(\mathbb{R}_{+}^{*} \times \Omega, \mathbb{R}\right)$.

ii. $\vartheta_{-}\left(\cdot, \mathbf{u}_{L}\right) \in \mathscr{C}^{2}\left(\left(0, \tau_{2}^{*}\right] \cup\left[\tau_{1}^{*}, \infty\right)\right)$.

iii. In $\left(0, \tau_{2}^{*}\right] \cup\left[\tau_{1}^{*}, \infty\right)$, the function $\vartheta_{-}\left(\cdot, \mathbf{u}_{L}\right)$ is strictly concave and strictly increasing.

iv. $\lim _{\tau \rightarrow 0^{+}} \vartheta_{-}\left(\tau, \mathbf{u}_{L}\right)=-\infty$ and

- If (H6a) holds, $\lim _{\tau \rightarrow \infty} \vartheta_{-}\left(\tau, \mathbf{u}_{L}\right)=+\infty$.

- If (H6b) holds, there exists a finite real constant $u_{\infty}^{-}\left(\mathbf{u}_{L}\right)$ such that $\lim _{\tau \rightarrow \infty} \vartheta_{-}\left(\tau, \mathbf{u}_{L}\right)=u_{\infty}^{-}\left(\mathbf{u}_{L}\right)$.

v. $\vartheta_{-}\left(\tau, \mathbf{u}_{L}\right)=\vartheta_{-}\left(\tau_{2}^{*}, \mathbf{u}_{L}\right)=\vartheta_{-}\left(\tau_{1}^{*}, \mathbf{u}_{L}\right)$ for all $\tau \in\left[\tau_{2}^{*}, \tau_{1}^{*}\right]$.
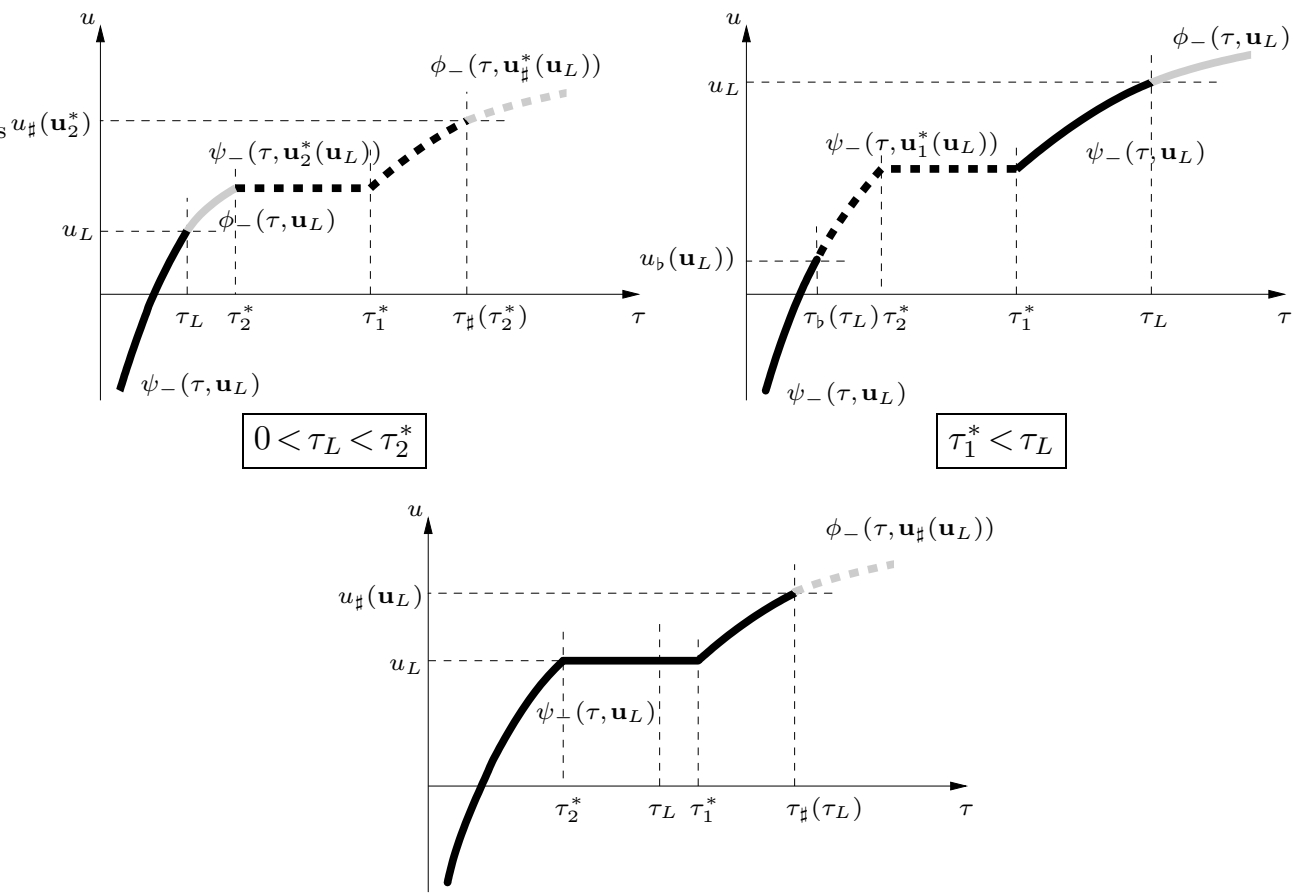

$\tau_{2}^{*} \leq \tau_{L} \leq \tau_{1}^{*}$

FIG. 4.3. The curve $\mathscr{U}_{-}\left(\mathbf{u}_{L}\right)$ according to the position of $\mathbf{u}_{L}$.

The proof of this proposition is given in Appendix $\mathrm{A}$ and the curve $\mathscr{U}_{-}\left(\mathbf{u}_{L}\right)$ is plotted in Fig. 4.3 (see also (A.2), (A.3) and (A.4) in the Appendix for explicit definitions of $\vartheta_{-}$). 
REMARK 4.4. Actually, the composition of the multiple waves, that is to say the nature of the waves which permit to connect any state $\overline{\mathbf{u}}$ to a given one $\mathbf{u}_{L}$, can easily be deduced from a geometric construction (see [30]). Assume that $\tau_{L}<\bar{\tau}$ and define $\check{\mathscr{P}}$ as the greatest convex function smaller or equal to $\mathscr{P}$. It is composed by affine and strictly convex parts. If we note $\tau_{a}$ and $\tau_{b}$ the limits of an affine part, then $\tau_{a}$ and $\tau_{b}$ are separated by a discontinuity. On the other hand, if $\tau_{a}$ and $\tau_{b}$ limit a strictly convex part $\check{\mathscr{P}}$, then $\tau_{a}$ and $\tau_{b}$ are separated by a rarefaction wave. The case $\bar{\tau}<\tau_{L}$ is similar, using the smallest concave function greater or equal to $\mathscr{P}$. Such a geometric characterization is classical in the scalar case, for a nonconvex flux [12].

\section{Resolution of the Riemann problem}

In order to solve the Riemann problem (1.1-1.4), we first present the set of nonnegative multiple waves $\mathscr{U}_{+}\left(\mathbf{u}_{R}\right)$. Afterwards, the solution of the Riemann problem is constructed, intersecting the sets $\mathscr{U}_{-}\left(\mathbf{u}_{L}\right)$ and $\mathscr{U}_{+}\left(\mathbf{u}_{R}\right)$ and the properties of the solution are investigated.

5.1. Nonnegative multiple waves. First, we must construct the set $\mathscr{U}_{+}\left(\mathbf{u}_{R}\right) \subset \Omega$, the analogue of $\mathscr{U}_{-}\left(\mathbf{u}_{L}\right)$, defined by

$$
\mathscr{U}_{+}\left(\mathbf{u}_{R}\right)=\left\{\mathscr{W}\left(0^{-} ; \mathbf{u}, \mathbf{u}_{R}\right), \mathbf{u} \in \Omega\right\}
$$

where $\mathscr{W}\left(x / t ; \mathbf{u}_{L}, \mathbf{u}_{R}\right)$ is a self-similar solution of the Riemann problem (1.1-1.4). Using the Galilean invariance of system (1.1-1.3), we directly obtain

$$
\mathscr{U}_{+}\left(\mathbf{u}_{R}\right)=\left\{(\tau, u) \in \Omega,\left(\tau, 2 u_{R}-u\right) \in \mathscr{U}_{-}\left(\mathbf{u}_{R}\right)\right\} .
$$

As above, $\vartheta_{+}$will denote the function defined by

$$
\begin{aligned}
\vartheta_{+}: \mathbb{R}_{+}^{*} \times \Omega & \longmapsto \mathbb{R} \\
\left(\tau, \mathbf{u}_{R}\right) & \longmapsto 2 u_{R}-\vartheta_{-}\left(\tau, \mathbf{u}_{R}\right) .
\end{aligned}
$$

Using simple calculations and Prop. 4.3, one may check:

Proposition 5.1. The function $\vartheta_{+}$satisfies for all $\mathbf{u}_{R} \in \Omega$ :

i. $\vartheta_{+} \in \mathscr{C}^{0}\left(\mathbb{R}_{+}^{*} \times \Omega, \mathbb{R}\right)$.

ii. $\vartheta_{+}\left(\cdot, \mathbf{u}_{R}\right) \in \mathscr{C}^{2}\left(\left(0, \tau_{2}^{*}\right] \cup\left[\tau_{1}^{*}, \infty\right)\right)$.

iii. In $\left(0, \tau_{2}^{*}\right] \cup\left[\tau_{1}^{*}, \infty\right)$, the function $\vartheta_{+}\left(\cdot, \mathbf{u}_{R}\right)$ is strictly convex and strictly decreasing.

iv. $\lim _{\tau \rightarrow 0^{+}} \vartheta_{+}\left(\tau, \mathbf{u}_{R}\right)=+\infty$ and

- If (H6a) holds, $\lim _{\tau \rightarrow \infty} \vartheta_{+}\left(\tau, \mathbf{u}_{R}\right)=-\infty$.

- If (H6b) holds, there exists a finite real constant $u_{\infty}^{+}\left(\mathbf{u}_{R}\right)$ such that $\lim _{\tau \rightarrow \infty} \vartheta_{+}\left(\tau, \mathbf{u}_{R}\right)=u_{\infty}^{+}\left(\mathbf{u}_{R}\right)$.

v. $\vartheta_{+}\left(\tau, \mathbf{u}_{R}\right)=\vartheta_{+}\left(\tau_{2}^{*}, \mathbf{u}_{R}\right)=\vartheta_{+}\left(\tau_{1}^{*}, \mathbf{u}_{R}\right)$ for all $\tau \in\left[\tau_{2}^{*}, \tau_{1}^{*}\right]$.

We turn now to the existence result for the solution of the Riemann problem $(1.1-1.4)$.

5.2. Existence of a solution. The existence of a solution is given by studying the set

$$
\mathscr{I}\left(\mathbf{u}_{L}, \mathbf{u}_{R}\right)=\mathscr{U}_{-}\left(\mathbf{u}_{L}\right) \cap \mathscr{U}_{+}\left(\mathbf{u}_{R}\right)
$$

More precisely, the solution of the Riemann problem (1.1-1.4) exists as soon as the set $\mathscr{I}$ is non empty. 
Lemma 5.2. If hypothesis (H6a) holds, the set $\mathscr{I}\left(\mathbf{u}_{L}, \mathbf{u}_{R}\right)$ is non empty for all $\mathbf{u}_{L}, \mathbf{u}_{R} \in \Omega$.

If hypothesis (H6b) holds, the set $\mathscr{I}\left(\mathbf{u}_{L}, \mathbf{u}_{R}\right)$ may be empty for some initial conditions $\mathbf{u}_{L}, \mathbf{u}_{R} \in \Omega$. In such a case, the solution of the Riemann problem (1.1-1.4) is completed with the use of the vacuum state $\{\tau=+\infty\}$.

Proof. Let us recall that under hypotheses $(\mathrm{H} 1-5)$, the function $\vartheta_{-}\left(\cdot, \mathbf{u}_{L}\right)$ is continuous, nonincreasing and $\lim _{\tau \rightarrow 0^{+}} \vartheta_{-}\left(\tau, \mathbf{u}_{L}\right)=-\infty$ (Prop. 4.3). In the same way, the function $\vartheta_{+}\left(\cdot, \mathbf{u}_{R}\right)$ is continuous, nondecreasing and we have $\lim _{\tau \rightarrow 0^{+}} \vartheta_{+}\left(\tau, \mathbf{u}_{R}\right)=$ $+\infty$ (Prop. 5.1).

If hypothesis (H6a) is fulfilled, we also have $\lim _{\tau \rightarrow \infty} \vartheta_{-}\left(\tau, \mathbf{u}_{L}\right)=+\infty$ and $\lim _{\tau \rightarrow \infty} \vartheta_{+}\left(\tau, \mathbf{u}_{R}\right)=-\infty$. Therefore, for any $\mathbf{u}_{L}, \mathbf{u}_{R} \in \Omega$, the graphs of $\vartheta_{-}\left(\cdot, \mathbf{u}_{L}\right)$ and $\vartheta_{+}\left(\cdot, \mathbf{u}_{R}\right)$ cross each other and thus $\mathscr{I}\left(\mathbf{u}_{L}, \mathbf{u}_{R}\right) \neq \emptyset$.

In the case of hypothesis (H6b), the functions $\vartheta_{-}\left(\cdot, \mathbf{u}_{L}\right)$ and $\vartheta_{+}\left(\cdot, \mathbf{u}_{R}\right)$ admit an asymptote when $\tau$ goes to $+\infty$ (see Props. 4.3 and 5.1). If $\mathbf{u}_{L}$ and $\mathbf{u}_{R}$ are such that $u_{\infty}^{-}\left(\mathbf{u}_{L}\right) \leq u_{\infty}^{+}\left(\mathbf{u}_{R}\right)$, the two graphs never meet and $\mathscr{I}\left(\mathbf{u}_{L}, \mathbf{u}_{R}\right)=\emptyset$. Therefore, the vacuum state $\{\tau=+\infty\}$ is introduced in order to connect the two graphs, as done in $[23,28,13]$ for instance.

As a consequence, this Lemma provides the existence result for the Riemann problem (1.1-1.4), allowing the use of the vacuum state when $u_{\infty}^{-}\left(\mathbf{u}_{L}\right) \leq u_{\infty}^{+}\left(\mathbf{u}_{R}\right)$.

REMARK 5.3. Let us note that $u_{\infty}^{-}\left(\mathbf{u}_{L}\right)$ (respectively $u_{\infty}^{+}\left(\mathbf{u}_{R}\right)$ ) can be made explicit, according to the position of $\mathbf{u}_{L}$ (resp. $\mathbf{u}_{R}$ ), using $u_{\infty}$, defined in (4.2). We thus can make explicit the conditions on $\mathbf{u}_{L}$ and $\mathbf{u}_{R}$ which yield the occurrence of the vacuum state.

5.3. Uniqueness of the solution. The uniqueness of the solution of the Riemann problem (1.1-1.4) would be obvious if, for any initial data $\mathbf{u}_{L}, \mathbf{u}_{R} \in \Omega$, the set $\mathscr{I}\left(\mathbf{u}_{L}, \mathbf{u}_{R}\right)$ was reduced to a singleton (under the condition of nonoccurrence of the vacuum state). Actually, assuming $u_{\infty}^{-}\left(\mathbf{u}_{L}\right)>u_{\infty}^{+}\left(\mathbf{u}_{R}\right), \mathscr{I}\left(\mathbf{u}_{L}, \mathbf{u}_{R}\right)$ is a singleton in most cases if $\mathscr{P}$ verifies (H6b). In order to illustrate such a case, let us construct the solution of the Riemann problem (1.1-1.4), for a specific couple $\left(\mathbf{u}_{L}, \mathbf{u}_{R}\right)$, such that $\tau_{L}<\tau_{2}^{*}, \tau_{2}^{*} \leq \tau_{R} \leq \tau_{1}^{*}$ and $\mathscr{I}\left(\mathbf{u}_{L}, \mathbf{u}_{R}\right) \subset\left(\tau_{\sharp}\left(\tau_{1}^{*}\right) \times \mathbb{R}\right)$. The solution is plotted in Fig. 5.1.
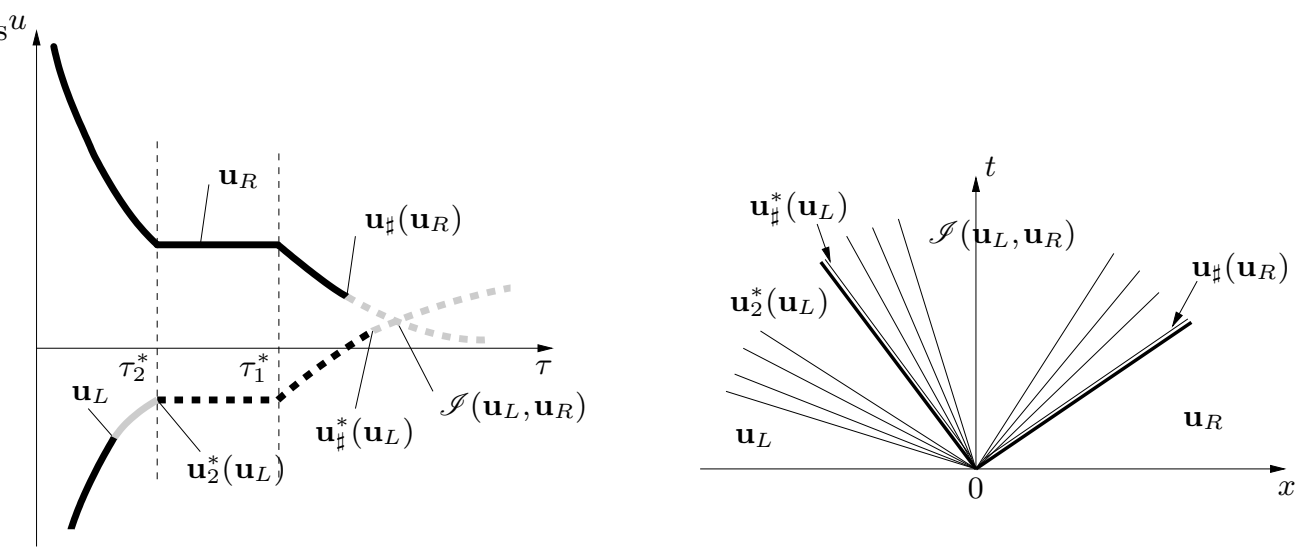

FIG. 5.1. An example of solution of the Riemann problem (1.1-1.4) in the ( $\tau, u)$ plane (left) and in the $(x, t)$ plane (right). 
It is composed by four constant states, separated by three rarefactions waves (two of them are attached with a discontinuity).

Actually, the set $\mathscr{I}\left(\mathbf{u}_{L}, \mathbf{u}_{R}\right)$ is a singleton except when $\mathbf{u}_{L}$ and $\mathbf{u}_{R}$ satisfy

$$
\vartheta_{-}\left(\tau_{2}^{*}, \mathbf{u}_{L}\right)=\vartheta_{+}\left(\tau_{2}^{*}, \mathbf{u}_{R}\right) .
$$

If equality (5.5) holds, the set $\mathscr{I}\left(\mathbf{u}_{L}, \mathbf{u}_{R}\right)$ is a segment of $\Omega$ :

$$
\mathscr{I}\left(\mathbf{u}_{L}, \mathbf{u}_{R}\right)=\left[\tau_{2}^{*}, \tau_{1}^{*}\right] \times\left\{\vartheta_{-}\left(\tau_{2}^{*}, \mathbf{u}_{L}\right)\right\}
$$

In such case, a careful study of the solutions must be performed.

Lemma 5.4. Let $\mathbf{u}_{L}, \mathbf{u}_{R} \in \Omega$ be such that (5.5) holds. Let us note $u_{0}=\vartheta_{-}\left(\tau_{2}^{*}, \mathbf{u}_{L}\right)$. Then the self-similar solution $\mathscr{W}\left(x / t ; \mathbf{u}_{L}, \mathbf{u}_{R}\right)$ of the Riemann problem (1.1-1.4) is unique and satisfies

- If $\tau_{L}<\tau_{2}^{*}$, then $\mathscr{W}\left(0^{-} ; \mathbf{u}_{L}, \mathbf{u}_{R}\right)=\left(\tau_{2}^{*}, u_{0}\right)$.

- If $\tau_{L}>\tau_{1}^{*}$, then $\mathscr{W}\left(0^{-} ; \mathbf{u}_{L}, \mathbf{u}_{R}\right)=\left(\tau_{1}^{*}, u_{0}\right)$.

- If $\tau_{L} \in\left[\tau_{2}^{*}, \tau_{1}^{*}\right]$, then $\mathscr{W}\left(0^{-} ; \mathbf{u}_{L}, \mathbf{u}_{R}\right)=\left(\tau_{L}, u_{0}\right)$.

- If $\tau_{R}<\tau_{2}^{*}$, then $\mathscr{W}\left(0^{+} ; \mathbf{u}_{L}, \mathbf{u}_{R}\right)=\left(\tau_{2}^{*}, u_{0}\right)$.

- If $\tau_{R}>\tau_{1}^{*}$, then $\mathscr{W}\left(0^{+} ; \mathbf{u}_{L}, \mathbf{u}_{R}\right)=\left(\tau_{1}^{*}, u_{0}\right)$.

- If $\tau_{R} \in\left[\tau_{2}^{*}, \tau_{1}^{*}\right]$, then $\mathscr{W}\left(0^{+} ; \mathbf{u}_{L}, \mathbf{u}_{R}\right)=\left(\tau_{R}, u_{0}\right)$.

Proof. As mentioned above, if $\mathbf{u}_{L}$ and $\mathbf{u}_{R}$ satisfy (5.5), then $\mathscr{I}\left(\mathbf{u}_{L}, \mathbf{u}_{R}\right)$ is given by Eq. (5.6). Consequently, there exists a fan, say $\xi_{l} \leq x / t \leq \xi_{r}$, where $\mathscr{W}\left(x / t ; \mathbf{u}_{L}, \mathbf{u}_{R}\right)$ can take any value in $\mathscr{I}\left(\mathbf{u}_{L}, \mathbf{u}_{R}\right)$ and thus might lead to the non-uniqueness of $\mathscr{W}\left(\cdot ; \mathbf{u}_{L}, \mathbf{u}_{R}\right)$

Let us look at such a solution. Assuming for instance that $\tau_{L}<\tau_{2}^{*}$, the definition of $\mathscr{U}_{-}\left(\mathbf{u}_{L}\right)$ (4.7) implies that $\mathscr{W}\left(\left(\xi_{l}\right)^{-} ; \mathbf{u}_{L}, \mathbf{u}_{R}\right)=\left(\tau_{2}^{*}, u_{0}\right)$. Let $\mathbf{u}_{l} \in \mathscr{I}\left(\mathbf{u}_{L}, \mathbf{u}_{R}\right)$ be the state directly connected to $\mathscr{W}\left(\left(\xi_{l}\right)^{-} ; \mathbf{u}_{L}, \mathbf{u}_{R}\right)$, that is to say $\mathbf{u}_{l}=\mathscr{W}\left(\left(\xi_{l}\right)^{+} ; \mathbf{u}_{L}, \mathbf{u}_{R}\right)$. By hypothesis (H4), the wave which connects $\mathscr{W}\left(\left(\xi_{l}\right)^{-} ; \mathbf{u}_{L}, \mathbf{u}_{R}\right)$ to $\mathbf{u}_{l}$ has a null speed and thus $\xi_{l}=0$. In the same way, assuming for instance $\tau_{R}<\tau_{2}^{*}$ provides $\xi_{r}=0$. Therefore, the fan of non-uniqueness is reduced to the line $x / t=0$ and $\mathscr{W}\left(0^{-} ; \mathbf{u}_{L}, \mathbf{u}_{R}\right)=\mathscr{W}\left(0^{+} ; \mathbf{u}_{L}, \mathbf{u}_{R}\right)=\left(\tau_{2}^{*}, u_{0}\right)$ (the solution is represented in Fig. 5.2). The
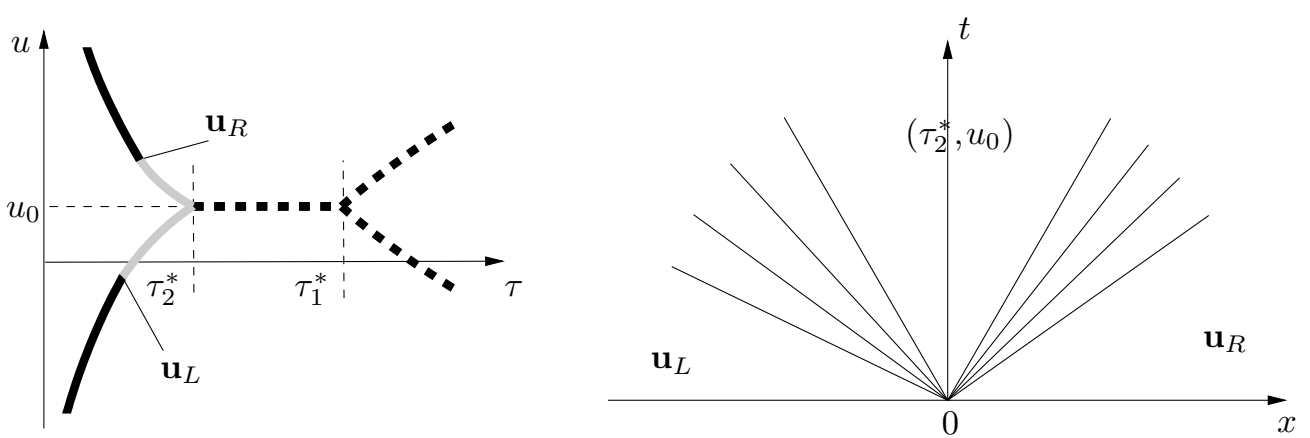

FIG. 5.2. The solution described in the proof of Lem. 5.4.

proof can easily be reproduced for the other positions of $\tau_{L}$ and $\tau_{R}$.

This Lemma concludes the proof of Theorem. 2.2. We now deal with the proof of Theorem. 2.3. 
5.4. Continuity with respect to initial data. We are interested here in the possible change of the structure of the self-similar solution $\mathscr{W}\left(x / t ; \mathbf{u}_{L}, \mathbf{u}_{R}\right)$ when changing the initial data $\mathbf{u}_{L}$ and $\mathbf{u}_{R}$.

When considering initial data $\mathbf{u}_{L}, \mathbf{u}_{R} \in \Omega$ such that $\mathscr{I}\left(\mathbf{u}_{L}, \mathbf{u}_{R}\right) \subset \Omega_{p}$, that is to say when the intersection between $\mathscr{U}_{-}\left(\mathbf{u}_{L}\right)$ and $\mathscr{U}_{+}\left(\mathbf{u}_{R}\right)$ is in a pure phase, the comparison result (2.4) is given by the continuity of $\vartheta_{-}$and $\vartheta_{+}$(see Prop. 4.3-i and Prop. 5.1-i) using classical arguments.

By contrast, the continuity in the neighborhood of a solution associated with initial data verifying (5.5) must be handled with care. We do not prove Thm. 2.3 in all the cases but we simply give an example of such initial data. The other delicate cases can be handled in the same spirit.

LEMmA 5.5. Let $\mathbf{u}_{L}$ and $\mathbf{u}_{R}$ be such that $\tau_{L}<\tau_{2}^{*}, \tau_{R}<\tau_{2}^{*}$ and such that (5.5) holds. Let $\mathbf{u}_{L}^{\varepsilon}=\left(\tau_{L}, u_{L}-\varepsilon\right)$. Then, for any $L>0$ and any $\varepsilon>0$ small enough, there exists a constant $C>0$ such that

$$
\int_{-L}^{L}\left|\mathscr{W}\left(\xi ; \mathbf{u}_{L}, \mathbf{u}_{R}\right)-\mathscr{W}\left(\xi ; \mathbf{u}_{L}^{\varepsilon}, \mathbf{u}_{R}\right)\right| d \xi \leq C \varepsilon
$$

Proof. Since $\mathbf{u}_{L}$ and $\mathbf{u}_{R}$ verify (5.5), we can define the velocity $u_{0}$ by

$$
u_{0}=\vartheta_{-}\left(\tau_{2}^{*}, \mathbf{u}_{L}\right)=\vartheta_{+}\left(\tau_{2}^{*}, \mathbf{u}_{R}\right) .
$$

Then, the self-similar solution $\mathscr{W}\left(\cdot ; \mathbf{u}_{L}, \mathbf{u}_{R}\right)$ is composed by two rarefaction waves (one has a negative speed and the other a positive speed) and the intermediate state $\mathbf{u}_{0}=\left(\tau_{2}^{*}, u_{0}\right)$.

Assuming now $\varepsilon>0$, we define

$$
\begin{aligned}
& \mathbf{u}_{1}^{\varepsilon}=\left(\tau_{1}^{\varepsilon}, u_{1}^{\varepsilon}\right)=\left(\tau_{2}^{*}, \vartheta_{-}\left(\tau_{2}^{*}, \mathbf{u}_{L}^{\varepsilon}\right)\right), \\
& \mathbf{u}_{2}^{\varepsilon}=\left(\tau_{2}^{\varepsilon}, u_{2}^{\varepsilon}\right)=\mathscr{I}\left(\mathbf{u}_{L}^{\varepsilon}, \mathbf{u}_{R}\right), \\
& \mathbf{u}_{3}=\left(\tau_{3}, u_{3}\right)=\left(\tau_{2}^{*}, \vartheta_{+}\left(\tau_{2}^{*}, \mathbf{u}_{R}\right)\right) .
\end{aligned}
$$

For any $\varepsilon>0$ small enough, the self-similar solution $\mathscr{W}\left(\cdot ; \mathbf{u}_{L}^{\varepsilon}, \mathbf{u}_{R}\right)$ is composed, from the left to the right, by a negative rarefaction wave between $\mathbf{u}_{L}^{\varepsilon}$ and $\mathbf{u}_{1}^{\varepsilon}$, a negative discontinuity between $\mathbf{u}_{1}^{\varepsilon}$ and $\mathbf{u}_{2}^{\varepsilon}$, a positive discontinuity between $\mathbf{u}_{2}^{\varepsilon}$ and $\mathbf{u}_{3}$ and a positive rarefaction wave between $\mathbf{u}_{3}$ and $\mathbf{u}_{R}$. All these waves are separated respectively by the constant states $\mathbf{u}_{1}^{\varepsilon}, \mathbf{u}_{2}^{\varepsilon}$ and $\mathbf{u}_{3}$. Note that the smallness of $\varepsilon$ is required in order to have $\tau_{2}^{\varepsilon}<\tau_{\sharp}\left(\tau_{2}^{*}\right)$ and avoid more complex wave patterns and, possibly, the occurrence of the vacuum state. Besides, we have $\mathbf{u}_{0}=\mathbf{u}_{3}$ (see Fig. 5.3).

We first study the difference $\mathscr{W}\left(\xi ; \mathbf{u}_{L}, \mathbf{u}_{R}\right)-\mathscr{W}\left(\xi ; \mathbf{u}_{L}^{\varepsilon}, \mathbf{u}_{R}\right)$ component by component, noting $\tau(\xi ; \mathbf{u}, \mathbf{v})$ and $u(\xi ; \mathbf{u}, \mathbf{v})$ the two components of $\mathscr{W}(\xi ; \mathbf{u}, \mathbf{v})$. By easy calculations, we can obtain more information about the velocity of the intermediate states:

$$
u_{1}^{\varepsilon}=u_{0}-\varepsilon \quad \text { and } \quad u_{0}-\varepsilon<u_{2}^{\varepsilon}<u_{0}
$$

Then, if we define

$$
\mathscr{A}_{u}=\int_{-L}^{L}\left|u\left(\xi ; \mathbf{u}_{L}, \mathbf{u}_{R}\right)-u\left(\xi ; \mathbf{u}_{L}^{\varepsilon}, \mathbf{u}_{R}\right)\right| d \xi,
$$



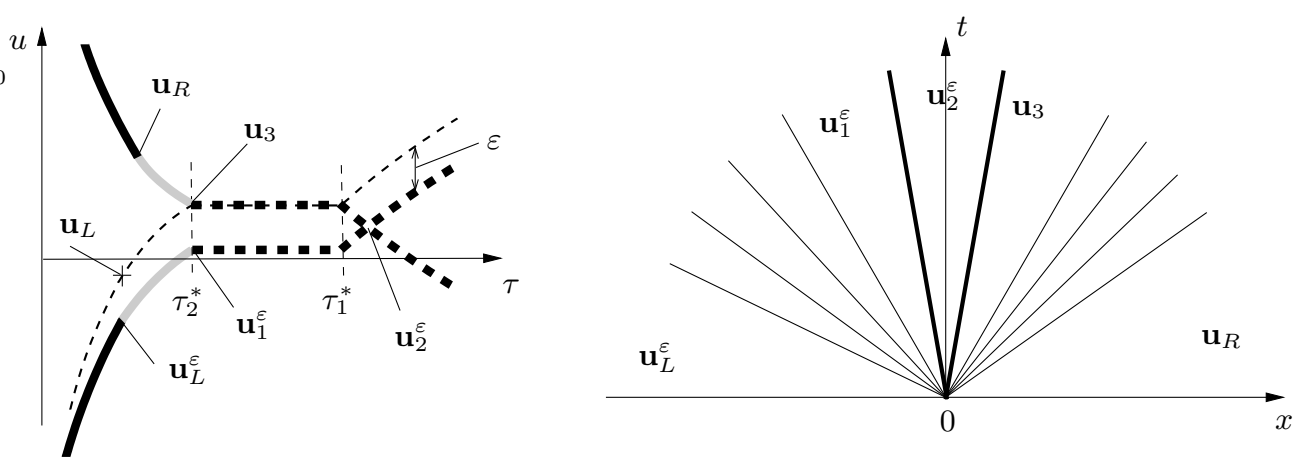

FIG. 5.3. The solution described in the proof of Lem. 5.5.

a coarse, but sufficient, estimate yields

$$
\begin{aligned}
\mathscr{A}_{u} & \leq \int_{-L}^{L}\left(u\left(\xi ; \mathbf{u}_{L}, \mathbf{u}_{R}\right)-\left(u\left(\xi ; \mathbf{u}_{L}, \mathbf{u}_{R}\right)-\varepsilon\right)\right) d \xi \\
& \leq 2 L \varepsilon .
\end{aligned}
$$

Let us focus now on the $\tau$-component of $\mathscr{W}\left(\xi ; \mathbf{u}_{L}, \mathbf{u}_{R}\right)-\mathscr{W}\left(\xi ; \mathbf{u}_{L}^{\varepsilon}, \mathbf{u}_{R}\right)$. The only difference between the two solutions is located between the two discontinuities, since $\mathbf{u}_{2}^{\varepsilon} \neq \mathbf{u}_{0}$. Then, defining

$$
\mathscr{A}_{\tau}=\int_{-L}^{L}\left|\tau\left(\xi ; \mathbf{u}_{L}, \mathbf{u}_{R}\right)-\tau\left(\xi ; \mathbf{u}_{L}^{\varepsilon}, \mathbf{u}_{R}\right)\right| d \xi,
$$

some manipulations based on the Rankine-Hugoniot jump relations and on $\tau_{2}^{\varepsilon}>\tau_{2}^{*}$ gives:

$$
\begin{aligned}
\mathscr{A}_{\tau} & \leq\left(\sigma\left(\mathbf{u}_{2}, \mathbf{u}_{3}\right)-\sigma\left(\mathbf{u}_{1}, \mathbf{u}_{2}\right)\right) \tau_{2}^{\varepsilon}, \\
& \leq\left(-\frac{u_{3}-u_{2}^{\varepsilon}}{\tau_{2}^{*}-\tau_{2}^{\varepsilon}}+\frac{u_{2}^{\varepsilon}-u_{1}^{\varepsilon}}{\tau_{2}^{\varepsilon}-\tau_{2}^{*}}\right) \tau_{2}^{\varepsilon}, \\
& \leq \frac{u_{3}-u_{1}^{\varepsilon}}{\tau_{2}^{\varepsilon}-\tau_{2}^{*}} \tau_{2}^{\varepsilon}, \\
& \leq\left(1+\frac{\tau_{2}^{*}}{\tau_{2}^{\varepsilon}-\tau_{2}^{*}}\right) \varepsilon, \\
& \leq\left(1+\frac{\tau_{2}^{*}}{\tau_{1}^{*}-\tau_{2}^{*}}\right) \varepsilon .
\end{aligned}
$$

Ineq. (5.7) thus results from the estimates on $\mathscr{A}_{u}$ and $\mathscr{A}_{\tau}$.

Note that in Lem. 5.5, we assumed that $\varepsilon$ is small whereas in Thm. 2.3 no assumption about the smallness of the initial data is stated. It could be understood as a contradiction. However, as mentioned in the proof of Lem. 5.5, the smallness of $\varepsilon$ relies on the occurrence of the self-similar solution given by $\left(\mathbf{u}_{L}, \mathbf{u}_{1}^{\varepsilon}, \mathbf{u}_{2}^{\varepsilon}, \mathbf{u}_{3}, \mathbf{u}_{R}\right)$. For greater $\varepsilon$, the solution becomes different but the continuity is then classical since $\mathscr{I}\left(\mathbf{u}_{L}, \mathbf{u}_{R}\right) \in \Omega_{p}$ (see the beginning of this section). In other words, Lem. 5.5 only provides a local result of continuity about initial data obeying (5.5) and the continuity 
for other initial data is given by classical arguments if $\mathscr{I}\left(\mathbf{u}_{L}, \mathbf{u}_{R}\right)$ is a singleton or by similar arguments as those of the above proof if (5.5) holds. This finally leads to the global continuity result stated in Thm. 2.3.

REMARK 5.6. The comparison result of Thm. 2.3 provides the continuity of the operator which links the initial data to the solution, in the $\mathbf{L}_{\text {loc }}^{1}$ topology, with respect to the self-similar structure of the solution. Let us emphasize that, due to the presence of a degenerate part in the pressure law, such a result is not available in the $\mathbf{L}^{\infty}$ topology (it is classical when dealing with resonant problems [26]).

We have thus been able to prove the well-posedness of the Riemann problem (1.1-1.4): existence, uniqueness and $\mathbf{L}_{\text {loc }}^{1}$-continuous dependence of the solution with respect to the initial data.

As mentioned before, the assumptions on the pressure law $\mathscr{P}$ can be relaxed. Indeed, the same results hold even if the pressure law is only nonincreasing and if each interval where $\mathscr{P}$ is constant is bounded. The main difference between such a case and the present work is that the construction of multiple waves becomes much more difficult.

\section{Appendix A. Proof of Proposition 4.3.}

Properties $i i i$, $i v$ and $v$ of function $\vartheta_{-}$are derived from Eqs. (4.6), (4.7) and (4.8) and from the behavior of $\phi_{-}$and $\psi_{-}$.

In order to obtain Property $i i$, we must study the smoothness of the function $\vartheta_{-}\left(\cdot, \mathbf{u}_{L}\right)$ at the following points:

a. $\tau=\tau_{\sharp}\left(\tau_{L}\right)$ if $\tau_{2}^{*} \leq \tau_{L} \leq \tau_{1}^{*}$,

b. $\tau=\tau_{\sharp}\left(\tau_{2}^{*}\right)$ if $\tau_{L}<\tau_{2}^{*}$,

c. $\tau=\tau_{\mathrm{b}}\left(\tau_{L}\right)$ if $\tau_{1}^{*}<\tau_{L}$.

Let us simplify the notations and define

$$
s\left(\tau_{a}, \tau_{b}\right)=\frac{\mathscr{P}\left(\tau_{a}\right)-\mathscr{P}\left(\tau_{b}\right)}{\tau_{a}-\tau_{b}}
$$

We first assume that $\tau_{2}^{*} \leq \tau_{L} \leq \tau_{1}^{*}$ and focus on the first derivative $\partial_{\tau} \vartheta_{-}$for $\tau=\tau_{\sharp}\left(\tau_{L}\right)$. When $\tau_{L}<\tau<\tau_{\sharp}\left(\tau_{L}\right)$, we have

$$
\partial_{\tau} \vartheta_{-}\left(\tau, \mathbf{u}_{L}\right)=\partial_{\tau} \psi_{-}\left(\tau, \mathbf{u}_{L}\right)=-\frac{1}{2}\left(\frac{\mathscr{P}^{\prime}(\tau)}{\sqrt{-s\left(\tau, \tau_{L}\right)}}-\sqrt{-s\left(\tau, \tau_{L}\right)}\right)
$$

Using the definition of $\tau_{\sharp}\left(\tau_{L}\right)$, we obtain

$$
\lim _{\tau \rightarrow \tau_{\sharp}\left(\tau_{L}\right)^{-}} \partial_{\tau} \vartheta_{-}\left(\tau, \mathbf{u}_{L}\right)=\sqrt{-\mathscr{P}^{\prime}\left(\tau_{\sharp}\left(\tau_{L}\right)\right)} .
$$

Now, if $\tau_{\sharp}\left(\tau_{L}\right)<\tau$, the derivative is given by

$$
\partial_{\tau} \vartheta_{-}\left(\tau, \mathbf{u}_{L}\right)=\partial_{\tau} \phi_{-}\left(\tau, \mathbf{u}_{L}\right)=\sqrt{-\mathscr{P}^{\prime}(\tau)}
$$

The first derivative $\partial_{\tau} \vartheta_{-}$is then continuous. Let us turn now to the second one, $\partial_{\tau \tau}^{2} \vartheta_{-}$. If $\tau_{1}^{*}<\tau<\tau_{\sharp}\left(\tau_{L}\right)$, some calculations give 


$$
\begin{aligned}
\partial_{\tau \tau}^{2} \vartheta_{-}\left(\tau, \mathbf{u}_{L}\right)= & \partial_{\tau \tau}^{2} \psi_{-}\left(\tau, \mathbf{u}_{L}\right), \\
= & \frac{1}{\left(\tau_{L}-\tau\right) \sqrt{-s\left(\tau_{L}, \tau\right)}}\left(\frac{\tau-\tau_{L}}{2} \mathscr{P}^{\prime \prime}(\tau)\right. \\
& \left.+\mathscr{P}^{\prime}(\tau)-\frac{\left(\mathscr{P}^{\prime}(\tau)+s\left(\tau_{L}, \tau\right)\right)^{2}}{4 s\left(\tau_{L}, \tau\right)}\right) .
\end{aligned}
$$

The definition of $\tau_{\sharp}\left(\tau_{L}\right)$ yields $s\left(\tau_{\sharp}\left(\tau_{L}\right), \tau_{L}\right)=\mathscr{P}^{\prime}\left(\tau_{\sharp}\left(\tau_{L}\right)\right)$. It leads to

$$
\lim _{\tau \rightarrow \tau_{\sharp}\left(\tau_{L}\right)^{-}} \partial_{\tau \tau}^{2} \vartheta_{-}\left(\tau_{\sharp}\left(\tau_{L}\right), \mathbf{u}_{L}\right)=\frac{-\mathscr{P}^{\prime \prime}\left(\tau_{\sharp}\left(\tau_{L}\right)\right)}{2 \sqrt{-\mathscr{P}^{\prime}\left(\tau_{\sharp}\left(\tau_{L}\right)\right)}} .
$$

On the other hand, if $\tau_{\sharp}\left(\tau_{L}\right)<\tau$, we have

$$
\partial_{\tau \tau}^{2} \vartheta_{-}\left(\tau, \mathbf{u}_{L}\right)=\partial_{\tau \tau}^{2} \phi_{-}\left(\tau, \mathbf{u}_{L}\right)=\frac{-\mathscr{P}^{\prime \prime}(\tau)}{2 \sqrt{-\mathscr{P}^{\prime}(\tau)}},
$$

which provides the continuity of the second derivative, replacing $\tau$ by $\tau_{\sharp}\left(\tau_{L}\right)$ in the previous equation, and concludes point a. Furthermore, the proof of the $\mathscr{C}^{2}$-continuity in the case $\mathrm{b}$ follows the same process.

We now focus on case c. If $\tau_{b}\left(\tau_{L}\right)<\tau<\tau_{2}^{*}$, the definition (4.8) leads to

$$
\partial_{\tau} \vartheta_{-}\left(\tau, \mathbf{u}_{L}\right)=\partial_{\tau} \psi_{-}\left(\tau, \mathbf{u}_{1}^{*}\left(\mathbf{u}_{L}\right)\right)=-\frac{1}{2}\left(\frac{\mathscr{P}^{\prime}(\tau)}{\sqrt{-s\left(\tau, \tau_{1}^{*}\right)}}-\sqrt{-s\left(\tau, \tau_{1}^{*}\right)}\right) .
$$

If $\tau<\tau_{b}\left(\tau_{L}\right)$, the derivative $\partial_{\tau} \vartheta_{-}$is given by

$$
\partial_{\tau} \vartheta_{-}\left(\tau, \mathbf{u}_{L}\right)=\partial_{\tau} \psi_{-}\left(\tau, \mathbf{u}_{L}\right)=-\frac{1}{2}\left(\frac{\mathscr{P}^{\prime}(\tau)}{\sqrt{-s\left(\tau, \tau_{L}\right)}}-\sqrt{-s\left(\tau, \tau_{L}\right)}\right) .
$$

By definition of $\tau_{b}\left(\tau_{L}\right)$, equality $s\left(\tau_{b}\left(\tau_{L}\right), \tau_{1}^{*}\right)=s\left(\tau_{b}\left(\tau_{L}\right), \tau_{L}\right)$ holds and ensures the continuity of $\partial_{\tau} \vartheta_{-}$, since

$$
\partial_{\tau} \psi_{-}\left(\tau_{b}\left(\tau_{L}\right), \mathbf{u}_{1}^{*}\left(\mathbf{u}_{L}\right)\right)=\partial_{\tau} \psi_{-}\left(\tau_{b}\left(\tau_{L}\right), \mathbf{u}_{L}\right) .
$$

Using now (A.1), we obtain

$$
\begin{aligned}
\lim _{\tau \rightarrow \tau_{b}\left(\tau_{L}\right)^{-}} \partial_{\tau \tau^{\prime}}^{2} \vartheta_{-}\left(\tau, \mathbf{u}_{L}\right) & =\partial_{\tau \tau}^{2} \psi_{-}\left(\tau_{b}\left(\tau_{L}\right), \mathbf{u}_{L}\right) \\
= & \frac{1}{\left(\tau_{L}-\tau_{b}\right) \sqrt{-s\left(\tau_{L}, \tau_{b}\right)}}\left(\frac{\tau_{b}-\tau_{L}}{2} \mathscr{P}^{\prime \prime}\left(\tau_{b}\right)\right. \\
& \left.+\mathscr{P}^{\prime}\left(\tau_{b}\right)-\frac{\left(\mathscr{P}^{\prime}\left(\tau_{b}\right)+s\left(\tau_{L}, \tau_{b}\right)\right)^{2}}{4 s\left(\tau_{L}, \tau_{b}\right)}\right),
\end{aligned}
$$

and

$$
\begin{aligned}
\lim _{\tau \rightarrow \tau_{b}\left(\tau_{L}\right)^{+}} \partial_{\tau \tau}^{2} \vartheta_{-}\left(\tau, \mathbf{u}_{1}^{*}\right) & =\partial_{\tau \tau}^{2} \psi_{-}\left(\tau_{b}\left(\tau_{L}\right), \mathbf{u}_{1}^{*}\right) \\
= & \frac{1}{\left(\tau_{1}^{*}-\tau_{b}\right) \sqrt{-s\left(\tau_{1}^{*}, \tau_{b}\right)}}\left(\frac{\tau_{b}-\tau_{1}^{*}}{2} \mathscr{P}^{\prime \prime}\left(\tau_{b}\right)\right. \\
& \left.\quad+\mathscr{P}^{\prime}\left(\tau_{b}\right)-\frac{\left(\mathscr{P}^{\prime}\left(\tau_{b}\right)+s\left(\tau_{1}^{*}, \tau_{b}\right)\right)^{2}}{4 s\left(\tau_{1}^{*}, \tau_{b}\right)}\right) .
\end{aligned}
$$


Here again, we use the definition of $\tau_{b}\left(\tau_{L}\right)$ which leads to the continuity of the second derivative:

$$
\lim _{\tau \rightarrow \tau_{b}\left(\tau_{L}\right)^{-}} \partial_{\tau \tau}^{2} \vartheta_{-}\left(\tau, \mathbf{u}_{L}\right)=\lim _{\tau \rightarrow \tau_{b}\left(\tau_{L}\right)^{+}} \partial_{\tau \tau}^{2} \vartheta_{-}\left(\tau, \mathbf{u}_{1}^{*}\right)
$$

This ends the proof of Property ii.

We now focus on the global continuity of $\vartheta_{-}$, stated in Property $i$. The continuity of $\vartheta_{-}$with respect to its first argument is ensured by Property $i i$ which we have just proved. It remains to prove the continuity of $\vartheta_{-}$with respect to $\mathbf{u}=\left(\tau_{L}, u_{L}\right)$. The behavior of $\vartheta_{-}$, or, in other words, the multiple structure of nonpositive waves, is deeply modified only when $\tau_{L}$ varies (in particular in the neighborhood of $\tau_{2}^{*}$ and $\tau_{1}^{*}$ ). Therefore, the continuity of $\vartheta_{-}$with respect to $u_{L}$ comes from the (classical property of) continuity of $\phi_{-}$and $\psi_{-}$with respect to $u_{L}$.

If we consider the continuity of $\vartheta_{-}$with respect to $\tau_{L}$, the only delicate points concern $\tau_{2}^{*}$ and $\tau_{1}^{*}$

Let us recall that if $\tau_{2}^{*} \leq \tau \leq \tau_{1}^{*}$, Eq. (4.6) yields

$$
\vartheta_{-}\left(\tau, \mathbf{u}_{L}\right)= \begin{cases}\psi_{-}\left(\tau, \mathbf{u}_{L}\right) & \text { if } 0<\tau<\tau_{L}, \\ u_{L} & \text { if } \tau=\tau_{L}, \\ \psi_{-}\left(\tau, \mathbf{u}_{L}\right) & \text { if } \tau_{L}<\tau \leq \tau_{\sharp}\left(\tau_{L}\right), \\ \phi_{-}\left(\tau, \mathbf{u}_{\sharp}\left(\mathbf{u}_{L}\right)\right) & \text { if } \tau_{\sharp}\left(\tau_{L}\right)<\tau .\end{cases}
$$

In the same way, for $0<\tau<\tau_{2}^{*}$, Eq. (4.7) gives

$$
\vartheta_{-}\left(\tau, \mathbf{u}_{L}\right)= \begin{cases}\psi_{-}\left(\tau, \mathbf{u}_{L}\right) & \text { if } 0<\tau<\tau_{L}, \\ u_{L} & \text { if } \tau=\tau_{L}, \\ \phi_{-}\left(\tau, \mathbf{u}_{L}\right) & \text { if } \tau_{L}<\tau \leq \tau_{2}^{*}, \\ \psi_{-}\left(\tau, \mathbf{u}_{2}^{*}\left(\mathbf{u}_{L}\right)\right) & \text { if } \tau_{2}^{*}<\tau \leq \tau_{\sharp}\left(\tau_{2}^{*}\right), \\ \phi_{-}\left(\tau, \mathbf{u}_{\sharp}\left(\mathbf{u}_{2}^{*}\left(\mathbf{u}_{L}\right)\right)\right) & \text { if } \tau_{\sharp}\left(\tau_{2}^{*}\right)<\tau,\end{cases}
$$

and, for $\tau_{1}^{*}<\tau$, Eq. (4.8) leads to

$$
\vartheta_{-}\left(\tau, \mathbf{u}_{L}\right)= \begin{cases}\psi_{-}\left(\tau, \mathbf{u}_{L}\right) & \text { if } 0<\tau<\tau_{b}\left(\tau_{L}\right), \\ \psi_{-}\left(\tau, \mathbf{u}_{1}^{*}\left(\mathbf{u}_{L}\right)\right) & \text { if } \tau_{b}\left(\tau_{L}\right) \leq \tau<\tau_{1}^{*}, \\ \psi_{-}\left(\tau, \mathbf{u}_{L}\right) & \text { if } \tau_{1}^{*} \leq \tau<\tau_{L}, \\ u_{L} & \text { if } \tau=\tau_{L} \\ \phi_{-}\left(\tau, \mathbf{u}_{L}\right) & \text { if } \tau_{L}<\tau .\end{cases}
$$

One can check that, letting $\tau_{L}$ go to $\tau_{2}^{*}$ in Eq. (A.3), the part $\phi_{-}\left(\tau, \mathbf{u}_{L}\right)$ for $\tau_{L}<\tau \leq \tau_{2}^{*}$ vanishes and $\mathbf{u}_{2}^{*}\left(\mathbf{u}_{L}\right)$ goes to $\mathbf{u}_{L}$. Eq. (A.3) then becomes Eq. (A.2) with $\tau_{L}=\tau_{2}^{*}$.

Likewise, when $\tau_{L} \rightarrow \tau_{1}^{*}$ in Eq. (A.4), then $\tau_{b}\left(\tau_{L}\right) \rightarrow 0$ and the part $\psi_{-}\left(\tau, \mathbf{u}_{L}\right)$ with $\tau \in\left[\tau_{1}^{*}, \tau_{L}\right)$ vanishes and since $\mathbf{u}_{1}^{*}\left(\mathbf{u}_{L}\right) \rightarrow \mathbf{u}_{L}$, for any $0<\tau<\tau_{L}$, the function $\vartheta_{-}\left(\tau, \mathbf{u}_{L}\right)$ becomes $\psi_{-}\left(\tau, \mathbf{u}_{L}\right)$ in (A.4). Moreover, setting $\tau_{L}=\tau_{1}^{*}$ in Eq. (A.2) gives $\tau_{\sharp}\left(\tau_{L}\right)=\tau_{1}^{*}$ (and $\mathbf{u}_{\sharp}\left(\mathbf{u}_{L}\right)=\mathbf{u}_{1}^{*}$ ). Therefore, Eq. (A.4) and Eq. (A.2) represent the same curves when $\tau_{L}=\tau_{1}^{*}$. It thus ensures the continuity of $\vartheta_{-}$with respect to $\tau_{L}$ and ends the proof of Proposition 4.3. 


\section{REFERENCES}

[1] F. Bachmann, Équations hyperboliques scalaires à flux discontinu, PhD thesis, Université de Provence, France, 2005.

[2] S. Benzoni-Gavage and H. Freistühler, Effects of surface tension on the stability of dynamical liquid-vapor interfaces, Arch. Ration. Mech. Anal., 174, 111-150, 2004.

[3] F. Bouchut, On zero pressure gas dynamics, in Advances in Kinetic Theory and Computing, in Ser. Adv. Math. Appl. Sci., World Sci. Publishing, River Edge, NJ, 22, 171-190, 1994.

[4] F. Bouchut and F. James, Duality solutions for pressureless gases, monotone scalar conservation laws, and uniqueness, Comm. Partial Differential Equations, 24, 2173-2189, 1999.

[5] F. Caro, F. Coquel, D. Jamet and S. Kokh, A simple finite-volume method for compressible isothermal two-phase flows simulation, Int. J. Finite Volumes, 3, 1-37, 2005.

[6] A. Chinnayya, A.-Y. Leroux and N. Seguin, A well-balanced numerical scheme for the approximation of the shallow-water equations with topography: the resonance phenomenon, Int. J. Finite Volumes, 1, 1-33, 2004.

[7] R. M. Colombo and F. S. Priuli, Characterization of Riemann solvers for the two phase psystem, Comm. Partial Differential Equations, 28, 1371-1389, 2003.

[8] C. M. Dafermos, The entropy rate admissibility criterion for solutions of hyperbolic conservation laws, J. Differential Equations, 14, 202-212, 1973.

[9] C. M. Dafermos, Hyperbolic conservation laws in continuum physics, Grundlehren der Mathematischen Wissenschaften [Fundamental Principles of Mathematical Sciences], SpringerVerlag, Berlin, 325, 2000.

[10] I. M. Gel'fand, Some problems in the theory of quasi-linear equations, Uspehi Mat. Nauk, 14, 87-158, 1959.

[11] P. Goatin and P. G. LeFloch, The Riemann problem for a class of resonant hyperbolic systems of balance laws, Ann. Inst. H. Poincaré Anal. Non Linéaire, 21, 881-902, 2004.

[12] E. Godlewski and P.-A. Raviart, Hyperbolic systems of conservation laws, Mathématiques \& Applications (Mathematics and Applications), Ellipses, Paris, 3(4), 1991.

[13] E. Godlewski and PA Raviart, Numerical Approximation of Hyperbolic Systems of Conservation Laws, Applied Mathematical Sciences, Springer-Verlag, New York, 1996.

[14] S. K. Godunov, A difference method for numerical calculation of discontinuous solutions of the equations of hydrodynamics, Mat. Sb. (N.S.), 47(89), 271-306, 1959.

[15] E. Isaacson, D. Marchesin, B. Plohr and B. Temple, The Riemann problem near a hyperbolic singularity: the classification of solutions of quadratic Riemann problems. I, SIAM J. Appl. Math., 48, 1009-1032, 1988.

[16] B. L. Keyfitz and H. C. Kranzer, The Riemann problem for a class of hyperbolic conservation laws exhibiting a parabolic degeneracy, J. Differential Equations, 47, 35-65, 1983.

[17] C. Klingenberg and N. H. Risebro, Convex conservation laws with discontinuous coefficients. Existence, uniqueness and asymptotic behavior, Comm. Partial Differential Equations, 20, 1959-1990, 1995.

[18] P. Krejčí and I. Straškraba, A uniqueness criterion for the Riemann problem, Hiroshima Math. J., 27, 307-346, 1997.

[19] P. G. LeFloch, Hyperbolic Systems of Conservation Laws, Lectures in Mathematics ETH Zürich, Birkhäuser Verlag, Basel, 2002.

[20] T. P. Liu, The entropy condition and the admissibility of shocks, J. Math. Anal. Appl., 53, 78-88, 1976.

[21] T. P. Liu, Resonance for quasilinear hyperbolic equation, Bull. Amer. Math. Soc. (N.S.), 6, 463-465, 1982.

[22] T.-P. Liu, Nonlinear resonance for quasilinear hyperbolic equation, J. Math. Phys., 28, 25932602, 1987.

[23] T. P. Liu and J. A. Smoller, On the vacuum state for the isentropic gas dynamics equations, Adv. in Appl. Math., 1, 345-359, 1980.

[24] V. Roytburd, The Riemann problem for a system of conservation laws modeling phase transitions, in Current Progress in Hyberbolic Systems: Riemann Problems and Computations (Brunswick, ME, 1988), of Contemp. Math., Amer. Math. Soc., Providence, RI, 100, 267276, 1989.

[25] V. Roytburd and M. Slemrod, An application of the method of compensated compactness to a problem in phase transitions, in Material Instabilities in Continuum Mechanics (Edinburgh, 1985-1986), Oxford Sci. Publ., Oxford Univ. Press, New York, 427-463, 1988.

[26] N. Seguin and J. Vovelle, Analysis and approximation of a scalar conservation law with a flux function with discontinuous coefficients, Math. Models Methods Appl. Sci., 13, 221-257, 2003. 
[27] M. Shearer, The Riemann problem for a class of conservation laws of mixed type, J. Differential Equations, 46, 426-443, 1982.

[28] J. Smoller, Shock Waves and Reaction-Diffusion Equations, Grundlehren der Mathematischen Wissenschaften (Fundamental Principles of Mathematical Science), Springer-Verlag, New York, 258, 1983.

[29] J. D. Towers, Convergence of a difference scheme for conservation laws with a discontinuous flux, SIAM J. Numer. Anal., 38, 681-698, 2000 (electronic).

[30] B. Wendroff, The Riemann problem for materials with nonconvex equations of state. I. Isentropic flow, J. Math. Anal. Appl., 38, 454-466, 1972. 